\title{
Eigen-transitions in cantilever cylindrical shells subjected to vertical edge loads
}

\author{
Ciprian D. Coman ${ }^{1 \dagger}$ and Andrew P. Bassom ${ }^{2}$ \\ ${ }^{1}$ School of Mathematical Sciences, University of Nottingham, \\ University Park, Nottingham NG7 2RD, UK \\ ${ }^{2}$ School of Physical Sciences, University of Tasmania, \\ Private Bag 37, Hobart TAS 7001, Australia
}

November 4, 2017

\begin{abstract}
A thin cantilever cylindrical shell subjected to a transverse shear force at the free end can experience two distinct modes of buckling, depending on its relative thickness and length. If the former parameter is fixed then a short cylinder buckles in a diffuse manner, while the eigenmodal deformation of a moderately long shell is localised, both axially and circumferentially, near its fixed end. Donnelltype buckling equations for cylindrical shells are here coupled with a non-symmetric membrane basic state to produce a linear boundary-value problem that is shown to capture the transition between the aforementioned instability modes. The main interest lies in exploring the approximate asymptotic separation of the independent variables in the corresponding stability equations, when the eigen-deformation is doubly localised. Comparisons with direct numerical simulations of the full buckling problem provide further insight into the accuracy and limitations of our approximations.
\end{abstract}

Keywords: cylindrical shells, localised buckling, shallow shell equations, multiple-scale asymptotics.

${ }^{\dagger}$ Corresponding author: cdc3p@yahoo.com 


\section{Introduction}

Thin-walled cylindrical shells are typically used as compression members in many practical applications. The elastic instability of such configurations when working in compression is very well known and has been the object of several excellent monographs (e.g., see $[1,2,3]$ ). However, compressive stresses in thin cylinders can also develop indirectly as a result of different loading conditions, such as pure bending or transverse edge forces. The elastic instabilities associated with these cases have received comparatively much less attention in the literature because they are not considered to be the primary loading modes for thin-walled cylinders. It was the relative scarcity of such results that prompted us to re-visit the buckling of a thin-walled cylinder loaded as a cantilever.

One of the earliest experimental studies dealing with the loss of stability in cantilever cylinders is that of Lundquist [4], who tested his samples under combined transverse shear and bending. His work revealed that there are essentially two distinct types of buckling mechanisms; one in which the tangential stresses in the shell predominate (shear or torsion mode), and another one governed by the action of the normal membrane stresses (bending mode). The corresponding two eigenmodes are shown schematically in Figure 1 for the case of a clamped shell. The former deformation pattern is spread out over the entire lateral surface of the cylinder and resembles that of a cylinder that buckles under the action of torsional loads, while the latter is strongly localised near the fixed end of the shell.
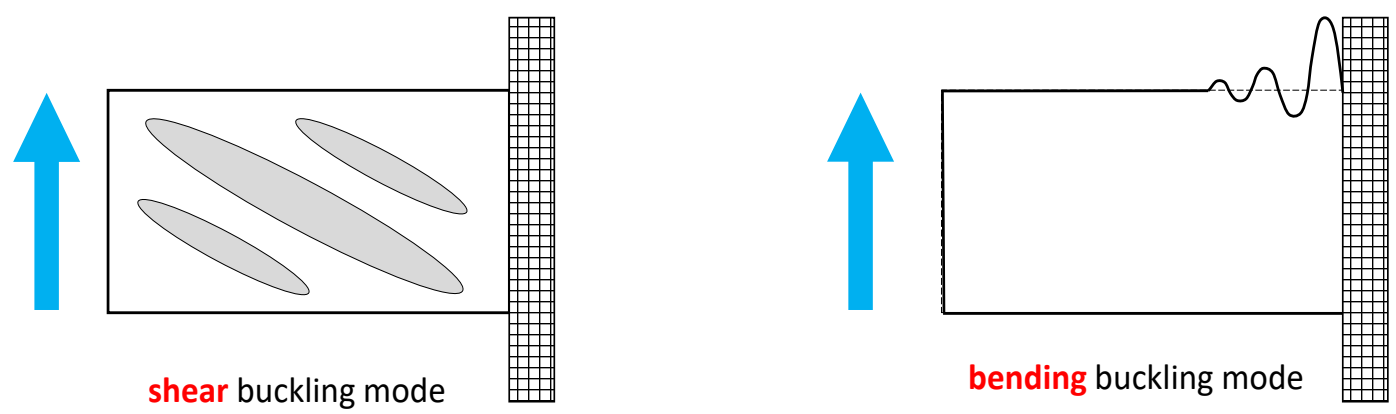

Figure 1: Two different buckling instability mechanisms of a cantilever circular cylindrical shell subjected to global vertical shear at the free end.

The absence of any rational/theoretical solutions for these two scenarios is not surprising, since even the simplest (linear) basic state for the above problem leads to principal stresses that vary nonuniformly, both axially and circumferentially. Some of the initial attempts $[5,6,7]$ to solve numerically the relevant buckling equations for the localised mode were based on rather crude, ad-hoc RayleighRitz approximations which, unsurprisingly, led to gross upper bounds for the critical load and should be regarded with suspicion. Schröder [8] seems to be the first who obtained a systematic numerical solution by using a double Fourier series, similar to the one employed in the current study. The accuracy of his results was later checked by Jamal [9] in a very interesting study that also accounted for the nonlinearity of the basic state, as well as different types of boundary conditions. Yamaki [1] used the same approach as Schröder to quantify the role of an additional hydrostatic pressure on the shear mode, and carried out extensive experiments regarding the post-buckling behaviour of cantilever cylinders. More recently, Manevich et al. $[10,11]$ have revisited the earlier experiments of Lundquist [4] for orthotropic thin cylindrical shells. They compared their experimental findings with a linear FE analysis obtained by using a commercial software package, and they found reasonable agreement between the two sets of data. 
Cantilever cylinders subjected to transverse forces have attracted much interest in relation to the design of liquid-storage tanks and large containment vessels. In this direction, it has long been known that under earthquake excitation the failure modes of these structures are strongly influenced by factors such as height-to-radius and thickness-to-radius ratios (e.g., see [12, 13, 14]). Hangai et al. [16] and Yoneda et al. [15] have been concerned with a simplified theoretical modelling of such problems in the static case, although their main interest lay in the ovalisation of the circular cross-section of the shell (e.g., [17]) and its role on triggering local buckling (see also [18, 19]). This ovalisation does have a profound effect on the location where the bending buckling mode is localised (cf. [16]); typically, the more pronounced the ovalisation is, the farther away from the fixed end will the localised mode be situated. Many other engineering aspects relevant to cylindrical storange tanks and their instabilities are discussed in [20] and [21].

In the present study we are motivated to explore the analogy between the localised (bending) mode mentioned above and the buckling pattern experienced by thin-walled cylinders in pure bending (e.g., see $[22,23,24])$. Although the similarity between the two scenarios has been known for a fairly long time (cf. [4]), no attempts have been made to treat these phenomena within a unifying theoretical framework. We take advantage of the recent works $[23,24]$ in which the pure bending problem was shown to be amenable to simple perturbation methods, both for linear and nonlinear basic states. Our aim here is to investigate the extent to which those asymptotic methods can be adapted to the global transverse shear setting. It must be stated right from the outset that the latter situation presents a significant complication. Unlike in the recent works just cited, even the linear (membrane) basic state exhibits dependence on both the axial and the hoop coordinates, and this fact precludes the direct use of solutions with separable variables. Although this key simplification is not currently available, it seems natural to inquire whether the independent variables can be separated asymptotically (at least in some approximate manner), and this is the main question we seek to address in this paper.

With this in mind, we start our study in $\S 2$ with a brief overview of the main bifurcation problem; these consist of the usual Donnell-Mushtari-Vlasov system coupled with the linear membrane solution for a cantilever isotropic cylinder subjected to a vertical shear force at the free end. It is shown in $\S 3$ that the basic state contains a great deal of information, and that one can actually predict qualitatively a number of important features regarding the two distinct buckling modes experienced by the cylinder. The properties of the full buckling equations are then illustrated in $\S 4$ by a representative sample of direct numerical simulations, which highlight the metamorphosis of the corresponding eigen-deformation as various parameters are varied. The next two sections deal with the specific details related to the (approximate) asymptotic separation of variables already mentioned. More precisely, in $\S 5$ we ignore the circumferential dependence to obtain a coupled system of ordinary differential equations in the axial variable only, and then pursue a simple double-scale asymptotic analysis. This information is later used in $\S 6$ to obtain another coupled differential system that is meant to approximate the localisation in the circumferential direction. Comparisons of the asymptotic approximations obtained with direct numerical simulations of the full eigenproblem for the cylinder provide further clues as to the accuracy and limitations of the strategy followed in this work. The paper concludes with a discussion of our results and some remarks on future work.

\section{The bifurcation equations}

We consider a thin cantilever cylindrical shell of length $L$, radius $R$ and uniform thickness $h(0<$ $h / R \ll 1)$ subjected to a transverse shear force at the free end, as indicated in Figure 2. The cylinder is assumed to be bounded by light bulkheads which are stiff in their own plane, but may be readily warped out of those plane. In what follows the cylinder is taken to be made of an isotropic elastic material characterised by the Young's modulus $E>0$ and the Poisson's ratio $0<\nu<1 / 2$.

The starting point for setting up the relevant bifurcation problem is the well-known Donnell- 
Mushtari-Vlasov (DMV) shallow-shell buckling equations (e.g., see [1, 25]) formulated in terms of the transverse displacement $w \equiv w(x, \theta)$ and a stress function $F \equiv F(x, \theta)$. If the scalar fields $\stackrel{\circ}{\sigma x}_{x x}$, $\stackrel{\circ}{\sigma}_{x \theta}$ and $\stackrel{\circ}{\sigma}_{\theta \theta}$ characterise the distribution of pre-buckling membrane stresses in the cylinder, then the aforementioned equations can be expressed as

$$
\begin{aligned}
& D \nabla^{4} w-h\left(\stackrel{\circ}{\sigma}_{x x} \frac{\partial^{2} w}{\partial x^{2}}+2 \stackrel{\circ}{x}_{x \theta} \frac{1}{R} \frac{\partial^{2} w}{\partial x \partial \theta}+\stackrel{\circ}{\sigma}_{\theta \theta} \frac{1}{R^{2}} \frac{\partial^{2} w}{\partial \theta^{2}}\right)-\frac{h}{R}\left(\frac{\partial^{2} F}{\partial x^{2}}\right)=0, \\
& \nabla^{4} F+\frac{E}{R}\left(\frac{\partial^{2} w}{\partial x^{2}}\right)=0, \quad \text { for } \quad 0 \leq x \leq L, \quad 0 \leq \theta \leq 2 \pi,
\end{aligned}
$$

where $D \equiv E h^{3} / 12\left(1-\nu^{2}\right)$ represents the bending rigidity and

$$
\nabla^{2} \equiv \frac{\partial^{2}}{\partial x^{2}}+\frac{1}{R^{2}} \frac{\partial^{2}}{\partial \theta^{2}}, \quad \nabla^{4}(\ldots)=\nabla^{2}\left[\nabla^{2}(\ldots)\right] .
$$

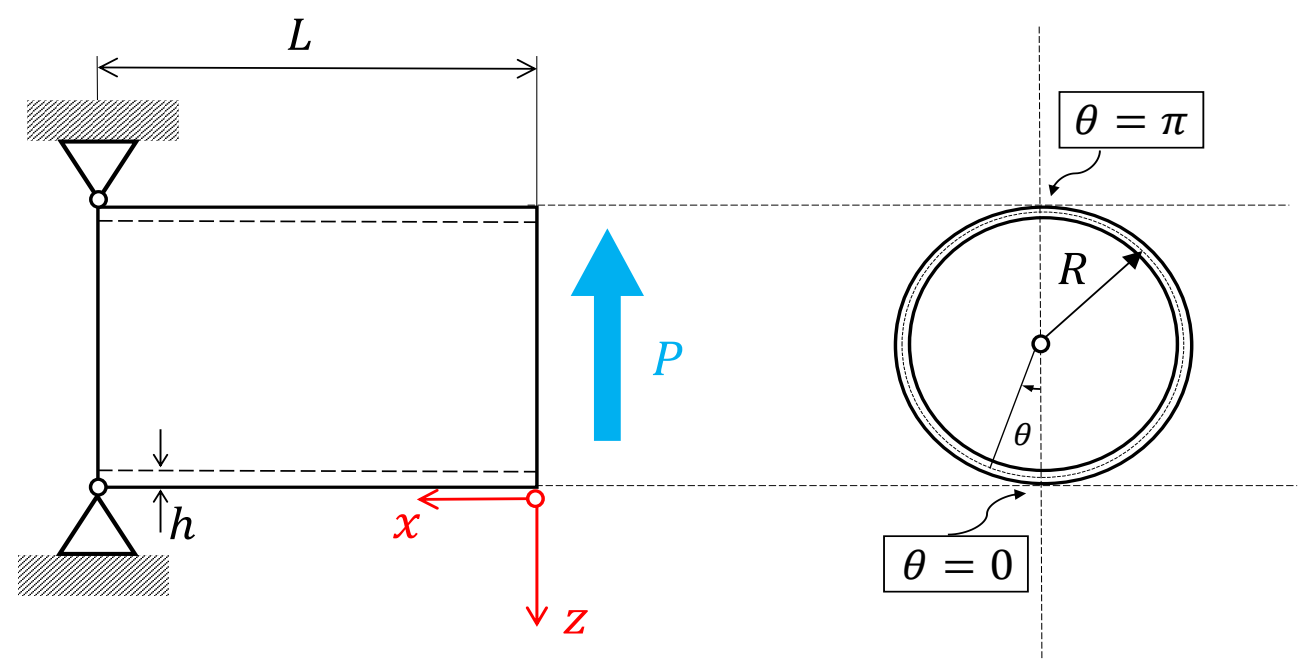

Figure 2: Simply supported cantilever circular cylindrical shell subjected to a global transverse shear force at the free end: side view (left) and details of the circular cross-section (right).

Following Schröder [8], prior to buckling the deformation of the cylindrical shell is taken to be described by a linear membrane state of stress. This leads to the non-symmetric closed-form solution for the in-plane stresses (e.g., see $[5,6]$ )

$$
\stackrel{\circ}{\sigma}_{x x}=\frac{P x}{\pi R^{2} h} \cos \theta, \quad \stackrel{\circ}{\sigma}_{x \theta}=-\frac{P}{\pi R h} \sin \theta, \quad \stackrel{\circ}{\sigma \theta}_{\theta \theta} \equiv 0 .
$$

On substituting (2.2) into (2.1), the resulting equations can be re-scaled by introducing the dimensionless quantities,

$$
\begin{aligned}
\bar{x}:=\frac{x}{L}, \quad \bar{w} & :=\frac{w}{R}, \quad \bar{F}:=12\left(1-\nu^{2}\right) \frac{F}{E h^{2}}, \quad \Lambda:=\frac{\sigma}{\sigma_{*}}, \\
\mu & :=\left[3\left(1-\nu^{2}\right)\right]^{1 / 2} \frac{R}{h}, \quad \alpha:=\frac{L}{R},
\end{aligned}
$$


where

$$
\sigma:=\frac{P}{\pi R h} \quad \text { and } \quad \sigma_{*}:=\frac{E}{\left[3\left(1-\nu^{2}\right)\right]^{1 / 2}}\left(\frac{h}{R}\right) .
$$

We note in passing that $\sigma_{*}$ represents the theoretical compressive buckling stress for infinitely long elastic cylindrical shells. Dropping the bars on the re-scaled variables, the non-dimensional bifurcation equations assume the form

$$
\begin{aligned}
& \nabla_{*}^{4} w-\alpha^{2} \frac{\partial^{2} F}{\partial x^{2}}-4 \mu \alpha^{3} \Lambda\left[x \cos \theta\left(\frac{\partial^{2} w}{\partial x^{2}}\right)-2 \sin \theta\left(\frac{\partial^{2} w}{\partial x \partial \theta}\right)\right]=0, \\
& \nabla_{*}^{4} F+4 \mu^{2} \alpha^{2} \frac{\partial^{2} w}{\partial x^{2}}=0, \quad \text { for } \quad 0 \leq x \leq 1, \quad 0 \leq \theta \leq 2 \pi,
\end{aligned}
$$

where the bi-Laplacian is now expressed as

$$
\nabla_{*}^{4} \equiv \frac{\partial^{4}}{\partial x^{4}}+2 \alpha^{2} \frac{\partial^{4}}{\partial x^{2} \partial \theta^{2}}+\alpha^{4} \frac{\partial^{4}}{\partial \theta^{4}} .
$$

The system (2.5) must be supplemented by appropriate boundary conditions. Here, we adopt simplysupported constraints at both the fixed and the free ends of the cylinder, that is

$$
w=\frac{\partial^{2} w}{\partial x^{2}}=F=\frac{\partial^{2} F}{\partial x^{2}}=0, \quad \text { for } \quad x \in\{0,1\} .
$$

In general, other types of boundary conditions would require the displacement version of the DMV equations (cf. [1]); we refer to Batdorf's discussion in [26] regarding the limitations that the $(w, F)$ system places on the implementation of various edge restraints (also, see [2]). In addition to (2.6), the transverse displacement and the stress function must be periodic in $\theta \in[0,2 \pi]$.

The motivation behind choosing the edge constraints (2.6) is twofold. First, simply-supported boundary conditions make the numerical analysis of the DMV shell theory relatively straightforward, as showed shortly in $\S 4$. Second, taken at face value, the use of a linear membrane pre-buckling stress state imposes some obvious limitations, which are intimately linked to the edge restraints. We take advantage of the extensive numerical study [9] that provides further insight into this particular aspect. In that work the stress distribution (2.2) was compared with a nonlinear general solution based on a Novozhilov-type shell theory [27], in which the geometric nonlinearity was restricted to small strains and moderate rotations (see also Sanders' discussion on pages 32-33 in [28]). It was found that for both short $(\alpha \simeq 2)$ and medium-length cylinders $(\alpha \simeq 6)$ the linear solution is a very good approximation if simply-supported boundary conditions are adopted. In the case when the free end of the shell $(x=1)$ is fully free, it was also confirmed that the membrane-stress approximation is still quite accurate for short cylinders, while significant deviations are encountered for medium-length and long shells. The origin of this discrepancy can be traced back to the pronounced ovalisation near the free end for the latter class of configurations. It is perhaps worth emphasising that, from a practical point of view, it is very difficult to achieve a fully free boundary condition while still applying the transverse shear force. Most of the experimental work reported in the literature has involved either simply-supported or clamped constraints at the free end (e.g., $[1,4,8]$ ).

\section{Some preliminary observations}

Before discussing the full numerical solution of the boundary-value problem (2.5)-(2.6) it is possible to gain some preliminary understanding of the behaviour of its solutions by examining closely the membrane pre-buckling stress distribution (2.2). Of course, the eigen-stresses that develop in the 
actual buckled cylinder are not the same, but the former are still indicative of the qualitative features of the buckling instability (e.g., see [29] for the case of a somewhat related case of a stretched annular plate subjected to uniform shear along its inner boundary).

We start by calculating the principal pre-buckling stresses, $\sigma_{1}$ and $\sigma_{2}$ (say), from (2.2); expressed in terms of the re-scaled variables (2.3) the final result is

$$
\sigma_{1,2}=\frac{1}{2} \sigma\left[\alpha x \cos \theta \pm\left(\alpha^{2} x^{2} \cos ^{2} \theta+4 \sin ^{2} \theta\right)^{1 / 2}\right],
$$

where $\sigma_{1}$ is taken to be the least of the two expressions obtained above. For reasons that will become transparent much later (in $\S 6$ ), we divide (3.1) by $\mu^{1 / 2}$ and let

$$
\delta:=\mu^{1 / 2} / \alpha .
$$

Figure 3 records a plot of the modified principal stresses $\widetilde{\sigma}_{j}:=2 \sigma_{j} /(\sigma \sqrt{\mu})$ for $R / h=50$ and $\delta=30$. This situation corresponds to the shear buckling of the cantilever cylinder; maximum compression and tension take place in two different sets of strips symmetrically placed on either side of the line $\theta=\pi$. If we consider the same information for $\delta=0.3$, as seen in Figure 4, it is clear that now the regions of compressive/tensile stresses change drastically, with the maximum compression being localised in the vicinity of the point

$$
\left(x_{0}, \theta_{0}\right) \equiv(1, \pi) .
$$

A less rigorous, but more direct, way of obtaining similar information as in Figures 3 and 4 is by plotting the expressions (3.2) for $0<\alpha \lesssim 1$ (shear buckling) and $\alpha \gtrsim 2$ (bending buckling).
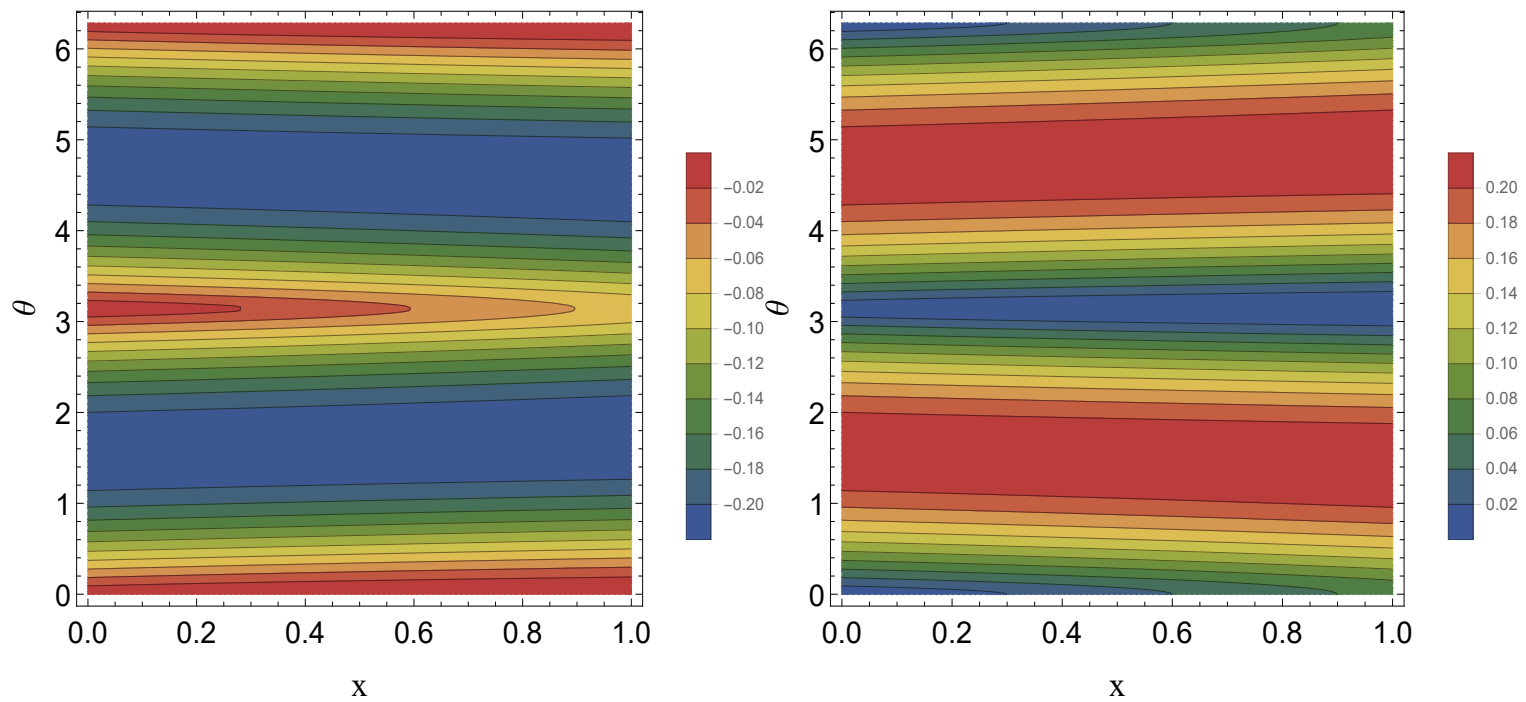

Figure 3: The distribution of the (modified) pre-buckling principal stresses $\widetilde{\sigma}_{1}$ (left) and $\widetilde{\sigma}_{2}$ (right), corresponding to $R / h=50$ and $\delta=30(\nu=0.3)$.

In their numerical study on the pure bending of thin cylinders Seide \& Weingarten [22] pointed out that the critical maximum membrane compressive stress was only slightly greater than that of an axially loaded shell, while Libai \& Durban [30] clarified that the two values should actually coincide in the limit $h \rightarrow 0$. A recent detailed study by the first author [23] provided more precise information about the rate of convergence of the two buckling values. In light of this evidence it seems reasonable to assume that, in a first approximation, bending buckling will occur when $\left|\sigma_{1}\right|=\sigma_{*}$ - see the definition 

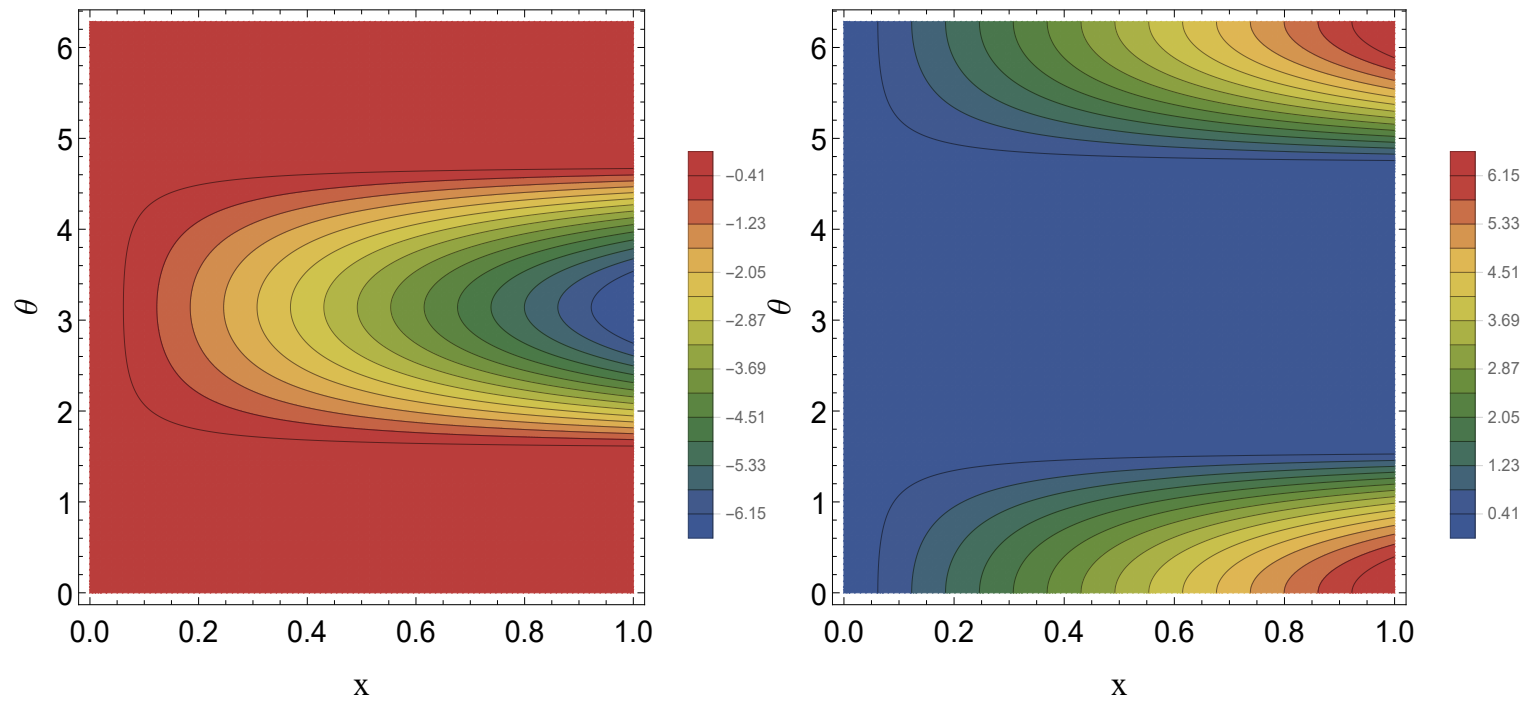

Figure 4: As per Fig. 3, except that here $\delta=0.3$.

(2.4), which leads to

$$
\Lambda f(x, \theta ; \alpha)=2, \quad \text { with } \quad f(x, \theta ; \alpha) \equiv\left(\alpha^{2} x^{2} \cos ^{2} \theta+4 \sin ^{2} \theta\right)^{1 / 2}-\alpha x \cos \theta .
$$

Noting that the maximum of the mapping $(x, \theta) \rightarrow f(x, \theta ; \alpha)$ is attained for $\left(x_{0}, \theta_{0}\right)$, we can immediately find a lower bound, $\Lambda_{\text {bend }}^{\dagger}$ (say), for the critical load value that is responsible for bending buckling, namely

$$
\Lambda_{\text {bend }}^{\dagger}=\frac{1}{\alpha} \text {. }
$$

This value is a fairly reasonable approximation for long cylinders $(\alpha>5)$. However, since for a fixed $R / h$ shorter configurations experience a rather different deformation pattern (cf. $[8,9]$ ), the above argument loses its validity and an alternative approach is required to deal with those cases. We remark briefly that it is possible to use the above information to estimate the shape of the region where the actual compressive stresses in the cylinder are less than $\left(-\sigma_{*}\right)$. For this, we write the buckling load as $\Lambda=\Lambda_{\text {bend }}^{\dagger}+\varepsilon$, where $0<\varepsilon \ll 1$ represents the collective contribution of the correction terms (in the actual bifurcation system scaling arguments suggest that $\varepsilon=\mathcal{O}\left(\mu^{-1 / 2}\right)$ ). The foregoing requirement translates into $\left(\Lambda_{\text {bend }}^{\dagger}+\varepsilon\right) f(x, \theta ; \alpha)-2>0$ which, for any fixed $\alpha>0$ can be regarded as a two-dimensional inequality in $(x, \theta)$. Its solution can be found routinely by using standard numerical methods, and we show in Figure 5 a sample of solution sets for several $\varepsilon$-values and $\alpha=6$. These sets correspond to the interior region of the semi-oval shapes adjacent to the fixed edge of the cylinder $(x=1)$.

The origin of the shear buckling pattern can also be traced back to the orientation of the principal stress directions (i.e, the eigenvectors associated with the eigenvalues $\sigma_{1}$ and $\sigma_{2}$ in (3.1)). Due to the dependence of the principal stresses on $(x, \theta)$, these eigen-directions will have a local character and are best visualised by plotting the stress trajectories (i.e., the "streamlines" of the two-dimensional membrane stress tensor). We recall that these geometric entities are found by integrating the differential equations

$$
\frac{d \theta}{d x}=-\frac{1}{2}\left(\frac{\stackrel{\circ}{\sigma}_{x x}}{\stackrel{\circ}{\sigma}_{x \theta}}\right) \pm \operatorname{sign}\left(\stackrel{\circ}{\sigma}_{x \theta}\right) \sqrt{\frac{\stackrel{\circ}{x x}_{x x}^{2}}{4 \circ_{x \theta}^{2}}+1}
$$




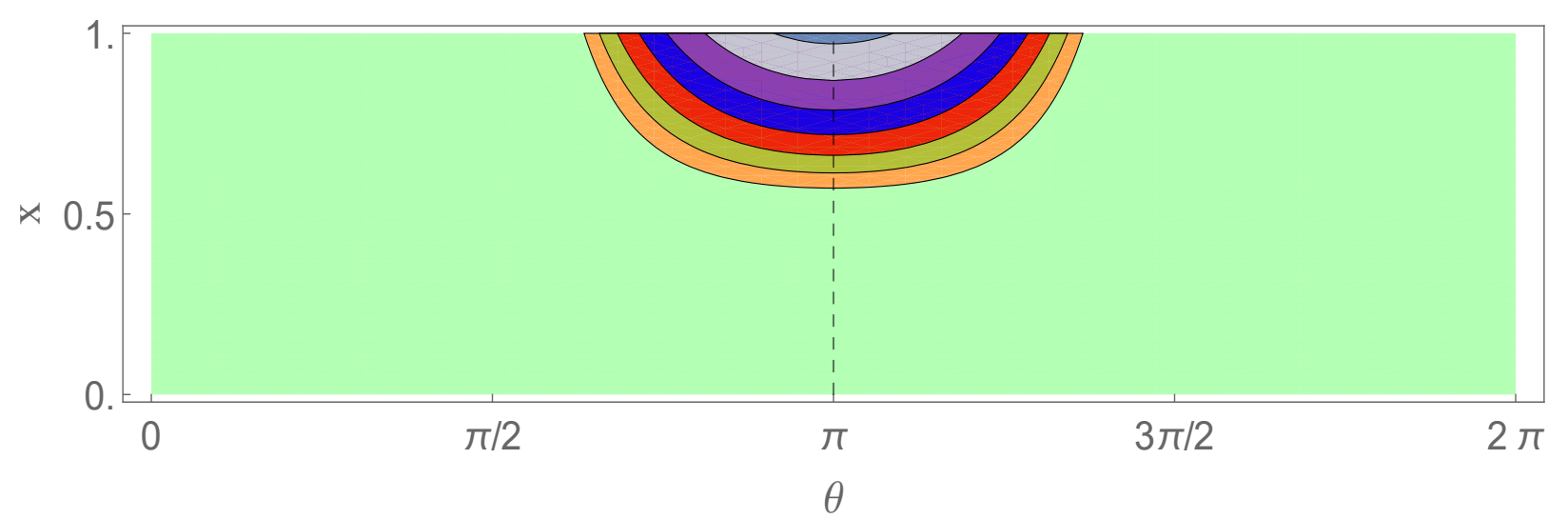

Figure 5: Examples of regions where $\left(\Lambda_{\text {bend }}^{\dagger}+\varepsilon\right) f(x, \theta ; \alpha)-2>0$, for $\varepsilon=(4 j+1) \times 10^{-2}$ and $j=0,1, \ldots, 6$. The size of these (semi-oval) regions grows with $\varepsilon$ - see text for further details.

where the 'minus' sign corresponds to the directions of tension $\left(\sigma_{2}\right)$ and the 'plus' sign gives the compressive directions. The solutions of (3.5) form two orthogonal networks of curves on the surface of the cylinder and, in general, through each point $(x, \theta) \in[0,1] \times[0,2 \pi]$ there pass two curves from each family. At particular locations where $\sigma_{1}=\sigma_{2}$ (if such points exist) there are an infinite number of stress trajectories passing through each of them. The stress trajectories for the pre-buckling stress tensor are illustrated in Figures 6 and 7 for the shear and bending buckling, respectively; to avoid overloading the discussion with unnecessary details the principal stresses are not modified as was done earlier in Figures 3 and 4. As we shall see shortly, in the next section, these pictures capture the qualitative nature of the buckling patterns quite faithfully. It should be kept in mind that this is just a rough picture of the instability mechanism - in reality the actual orientations of the general stress trajectories will be a small perturbation of the ones seen in the figures just mentioned.
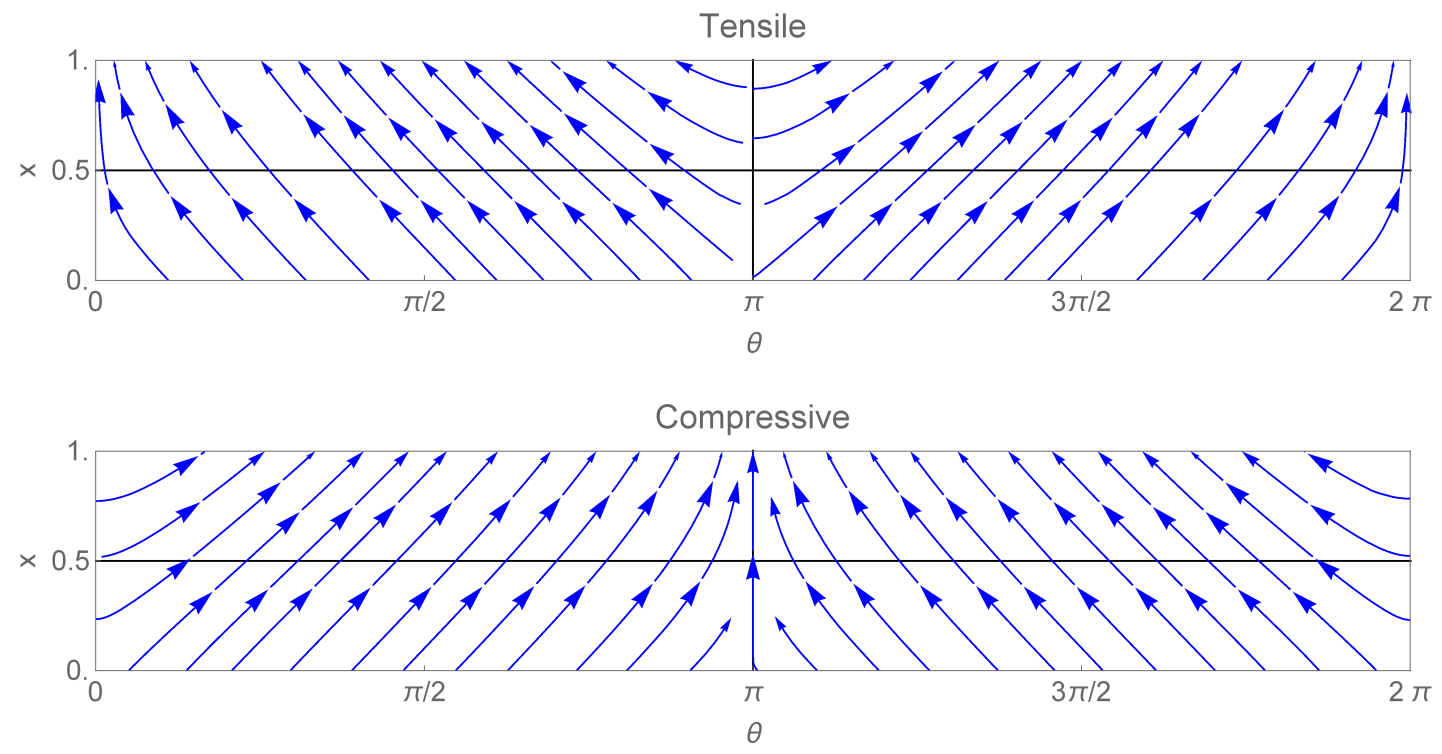

Figure 6: Stress trajectories for the membrane pre-buckling stress tensor (2.2). The upper window shows the directions along which the cylinder is being stretched; compression is experienced along the family of curves included in the lower window. These results are obtained from numerically integrating (3.5) for $\alpha=0.5$ and formally correspond to shear buckling. 

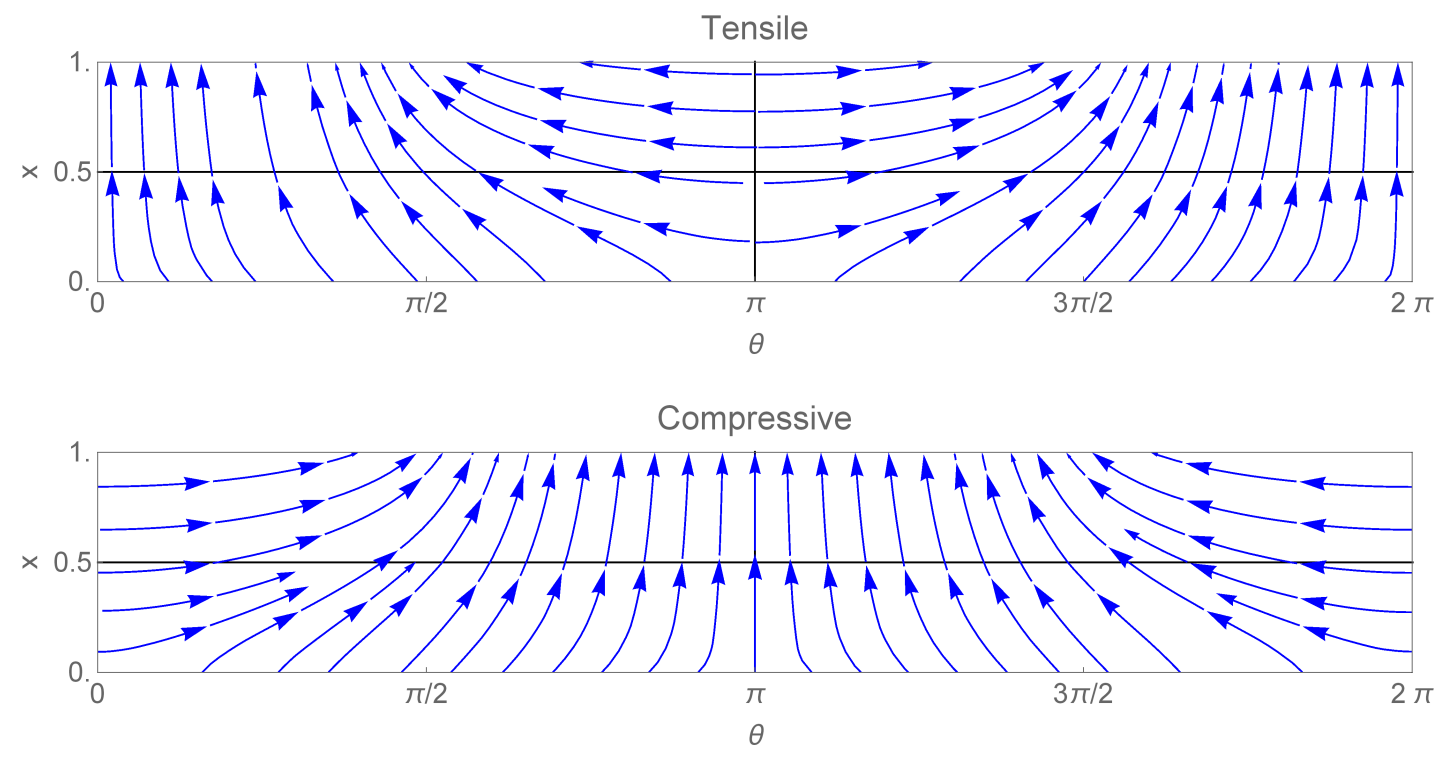

Figure 7: As per Fig. 6, except that here $\alpha=7.6$ (corresponding to bending buckling).

For short and moderately long circular cylindrical shells under the action of the torque $M$ applied at their ends, Donnell found (cf. [3]) that the critical shear stress can be approximated by the formula

$$
\left(1-\nu^{2}\right) \frac{\tau_{\mathrm{cr}}}{E}\left(\frac{L}{h}\right)^{2}=2.8+\sqrt{2.6+1.4\left(\sqrt{1-\nu^{2}} \frac{L^{2}}{2 R h}\right)^{3 / 2}}
$$

where $\tau:=M /\left(2 \pi R^{2} h\right)$ and $\tau_{\mathrm{cr}}$ denotes its lowest critical value associated with buckling. A quick look at our shear stresses in (2.2) indicates that their maximum is equal to $\sigma$. If we change $\tau \rightarrow \sigma$ in (3.6) and use the re-scaled variables of $\S 2$, it is found that a rather crude estimate for the critical load, $\Lambda_{\text {shear }}^{\dagger}$ (say), that leads to shear buckling in our configuration is given by

$$
\Lambda_{\text {shear }}^{\dagger}=\frac{3}{\mu \alpha^{2}}\left[\beta_{0}+\left(\beta_{1}+\beta_{2} \mu^{3 / 2} \alpha^{3}\right)^{1 / 2}\right]
$$

with $\beta_{0}:=2.8, \beta_{1}:=2.6$ and $\beta_{2}:=1.4 \cdot 2^{-3 / 2} 3^{-3 / 4} \simeq 0.22$. This formula can be employed to yield an approximation for the transition between shear and bending buckling. To this end, we simply equate the right-hand sides of (3.4) and (3.7) to get a cubic equation in $\alpha$, whose largest root has the asymptotic expansion $(\mu \gg 1)$

$$
\alpha=\frac{1}{9 \beta_{2}} \mu^{1 / 2}-6 \beta_{0} \mu^{-1}-81 \beta_{2}\left(\beta_{1}+3 \beta_{0}^{2}\right) \mu^{-5 / 2}-4374 \beta_{0} \beta_{2}^{2}\left(3 \beta_{1}+5 \beta_{0}^{2}\right) \mu^{-4}+\ldots ;
$$

the other two roots are $\mathcal{O}\left(\mu^{-1}\right)$ and are irrelevant for our discussion. For $R / h=100$ the expression (3.8) gives a transition point $\alpha_{c} \simeq 6.389$, which compares relatively well with our direct numerical simulations (as well as the previous numerical studies [8,9]). Thus, for $2<\alpha<\alpha_{c}$ we expect the buckling of the shell to be akin to torsional buckling of short cylinders, while for $\alpha>\alpha_{c}$ the deformation becomes localised near the point $\left(x_{0}, \theta_{0}\right)$ and will have a similar character to the buckling of a cylinder subjected to in-plane (or pure) bending. The two regimes are represented schematically in Figure 8.

Although the estimate we have just found can only be viewed as an orientative value, formula (3.8) does show that the transition between the two different buckling regimes occurs for $\alpha=\mathcal{O}\left(\mu^{1 / 2}\right)$. This 
has several implications, especially for the interpretation of the numerical results. Both Schröder [8] and Jamal [9] have considered fixed values of $R / h$ and they varied $\alpha$ to exhibit the buckling transition; this is equivalent to fixing the thickness of the cantilever cylinder and varying its length. In light of the foregoing discussion, and as confirmed by our direct numerical simulations, an equivalent approach would be to fix $\alpha$ and vary $\mu$. In this case there is a critical value of the latter parameter, $\mu_{c}$ (say), such that for $0<\mu<\mu_{c}$ the buckling pattern is localised near the fixed end of the cylinder, while for $\mu>\mu_{c}$ this eigen-deformation is diffuse and evocative of shear buckling for axially twisted short cylinders.

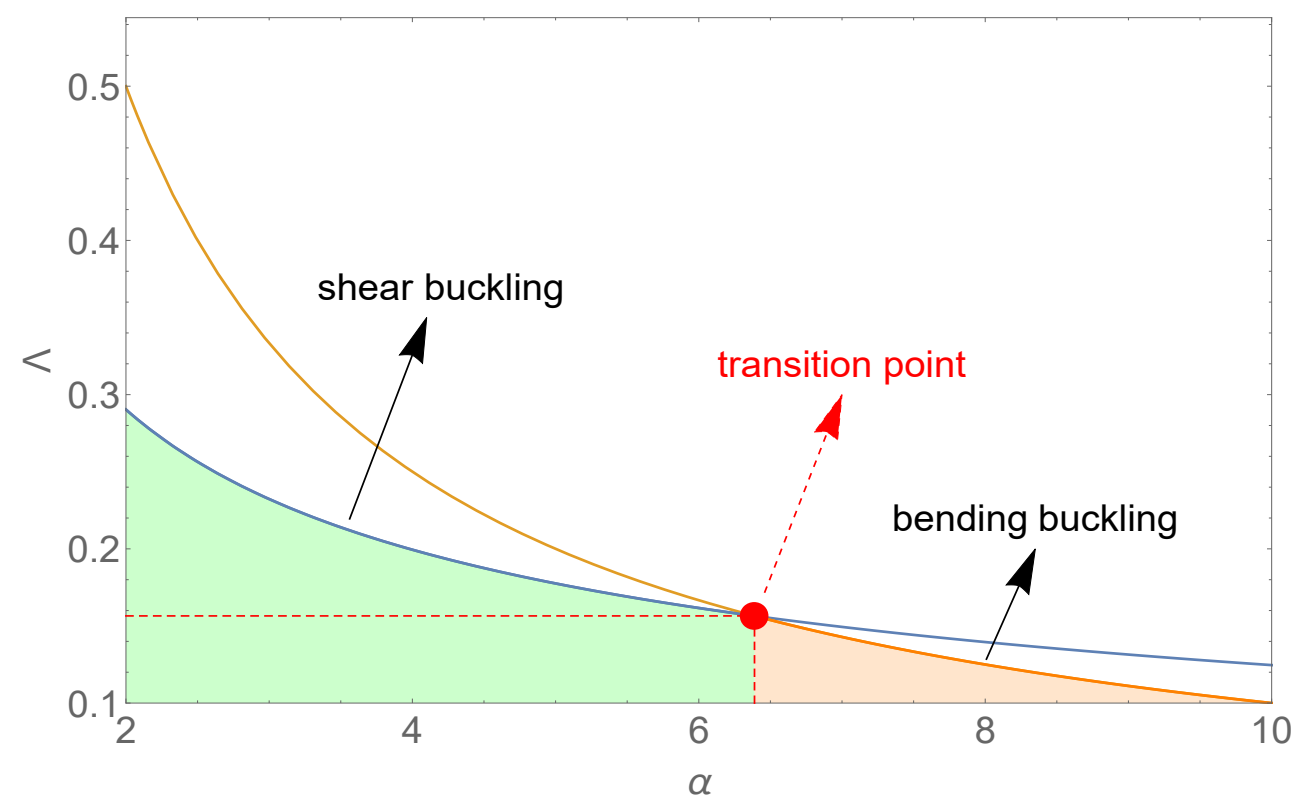

Figure 8: Illustration of the transition point (red marker) in the buckling of the cantilever shell subjected to vertical shear at the free end. The blue curve corresponds to the function $\Lambda=\Lambda_{\text {shear }}^{\dagger}(\alpha)$ defined in (3.7), while the orange curve represents the graph of $\Lambda=\lambda_{\text {bend }}^{\dagger}(\alpha)$ given by the simple expression (3.4). Here $R / h=100$.

\section{Numerical solutions}

The numerical solution of (2.5) subject to suitable boundary conditions can be found in a number of different ways. The most expedient approach is the strategy followed by Batdorf [26] for the classical buckling of axially compressed cylinders. Instead of reducing the system (2.5) to an eighth-order partial differential equation in $w$, he used the inverse of the bi-Laplacian in the compatibility equation (2.5b) to express $F$ in terms of the partial derivative of $w$, which was subsequently substituted in the equilibrium equation (2.5a). In the present context these formal manipulations lead to

$$
\mathcal{L}[w] \equiv \nabla_{*}^{4} w+4 \mu^{2} \alpha^{4} \nabla_{*}^{-4}\left(\frac{\partial^{4} w}{\partial x^{4}}\right)-4 \mu \alpha^{3} \Lambda\left[x \cos \theta\left(\frac{\partial^{2} w}{\partial x^{2}}\right)-2 \sin \theta\left(\frac{\partial^{2} w}{\partial x \partial \theta}\right)\right]=0 .
$$

If the ends of the cylinder are simply supported the solution of (4.1) can be found by using a global Galerkin method in conjunction with the suitably truncated double Fourier series,

$$
w(x, \theta)=\sum_{m=1}^{K} \sum_{n=1}^{K} a_{m n} \varphi_{m n}(x, \theta),
$$


where $a_{m n} \in \mathbb{R}$ are unknown coefficients (the degrees of freedom of the discretised shell) and

$$
\varphi_{m n}(x, \theta):=\sin \left(\lambda_{m} x\right) \cos [(n-1) \theta], \quad \lambda_{m}:=m \pi, \quad(m, n=1,2, \ldots, K) ;
$$

the number of terms in each sum in (4.2) is chosen so that the calculated value of $\Lambda$ converges, as explained shortly. The Galerkin method requires the following orthogonality conditions to be satisfied

$$
\sum_{m=1}^{K} \sum_{n=1}^{K} \int_{0}^{1} \int_{0}^{2 \pi} \mathcal{L}\left[\varphi_{m n}\right](x, \theta) \varphi_{p q}(x, \theta) \mathrm{d} x \mathrm{~d} \theta=0, \quad \text { for } \quad p, q=1,2, \ldots, K,
$$

which eventually yield an algebraic system of $K^{2}$ equations in $a_{m n}(1 \leq m, n \leq K)$,

$$
\sum_{m=1}^{K} \sum_{n=1}^{K} a_{m n} f(m, n) \Gamma_{m n p q}^{(1)}+4 \alpha^{-1} \Lambda \sum_{m=1}^{K} \sum_{n=1}^{K} a_{m n}\left[g(m) \Gamma_{m n p q}^{(2)}+h(m, n) \Gamma_{m n p q}^{(3)}\right]=0
$$

where

$$
\begin{gathered}
f(m, n):=\mu^{-1}\left[\left(\frac{\lambda_{m}}{\alpha}\right)^{2}+(n-1)^{2}\right]^{2}+4 \mu\left[1+\left(\frac{\alpha}{\lambda_{m}}\right)^{2}(n-1)^{2}\right]^{-2}, \\
g(m):=\lambda_{m}^{2}, \quad h(m, n):=-2(n-1) \lambda_{m}
\end{gathered}
$$

and $\Gamma_{m n p q}^{(j)}=B_{m p}^{(j)} C_{n q}^{(j)}(j=1,2,3)$, with

$$
\begin{array}{lll}
B_{m p}^{(1)}:=\int_{0}^{1} \sin \left(\lambda_{m} x\right) \sin \left(\lambda_{p} x\right) \mathrm{d} x, & C_{n q}^{(1)}:=\int_{0}^{2 \pi} \cos [(n-1) \theta] \cos [(q-1) \theta] \mathrm{d} \theta, \\
B_{m p}^{(2)}:=\int_{0}^{1} x \sin \left(\lambda_{m} x\right) \sin \left(\lambda_{p} x\right) \mathrm{d} x, & C_{n q}^{(2)}:=\int_{0}^{2 \pi} \cos \theta \cos [(n-1) \theta] \cos [(q-1) \theta] \mathrm{d} \theta, \\
B_{m p}^{(3)}:=\int_{0}^{1} \cos \left(\lambda_{m} x\right) \sin \left(\lambda_{p} x\right) \mathrm{d} x, & C_{n q}^{(3)}:=\int_{0}^{2 \pi} \sin \theta \sin [(n-1) \theta] \cos [(q-1) \theta] \mathrm{d} \theta ;
\end{array}
$$

these integrals can be evaluated either by using integration by parts or numerically.

If the unknowns $a_{m n}$ are collected together in a column vector $\boldsymbol{u}$, then the system (4.4) can be written in symbolic form as

$$
\left(\boldsymbol{A}+4 \alpha^{-1} \Lambda \boldsymbol{B}\right) \boldsymbol{u}=\mathbf{0},
$$

for some suitably defined $K \times K$ real matrices $\boldsymbol{A}$ and $\boldsymbol{B}$. Letting $\Lambda^{(K)}$ and $\Lambda^{(K+1)}$ be the calculated values of $\Lambda$ from solving the generalised eigenproblem (4.10) corresponding to the ansatz (4.2) with $K^{2}$ and $(K+1)^{2}$ terms, respectively, then the number of terms in the foregoing assumed expansion is chosen so that $\left|\Lambda^{(K+1)}-\Lambda^{(K)}\right|<10^{-s}$ for some fixed positive integer $s$; in the numerical simulations presented in this section we typically used $s=4$ and/or $s=5$.

For boundary conditions other than the simply-supported type the Galerkin method requires considering both equations in (2.5) and using an ansatz of the form

$$
w(x, \theta)=\sum_{n=1}^{K} a_{n}(\theta) \sin \left(\lambda_{m} x\right), \quad F(x, \theta)=\sum_{n=1}^{K} b_{n}(\theta) \sin \left(\lambda_{m} x\right),
$$

in which the unknown amplitudes $a_{n}(\theta), b_{n}(\theta)(n=1,2, \ldots, K)$ are to be found. Imposing the usual orthogonality conditions as explained above results in a system of $8 K$ first-order ordinary differential 
equations in $0 \leq \theta \leq 2 \pi$ for the aforementioned amplitudes, that is then solved numerically subject to appropriate periodicity conditions. In the interest of brevity we do not elaborate further on this strategy, although we have used both approaches to check our results.

Figure 9 illustrates the dependence of the loading parameter $\Lambda$ on $\mu$ for $\alpha=6$; this situation is representative for other values of $\alpha$ as well - Figure 10 contains two further examples corresponding to $\alpha=5$ and $\alpha=8$, respectively. The response curves $\Lambda=\Lambda(\mu ; \alpha)$ have several notable features that are identified explicitly in the former figure. For instance, as already anticipated by our discussion in $\S 3$, for a given $\alpha>0$ there is a critical value of $\mu=\mu_{c}$ that corresponds to a kink in the response curve; this marks the transition between bending buckling (to its left) and shear buckling (to its right). The kink is not sharp, but it has a very small radius of curvature. To appreciate the changes undergone

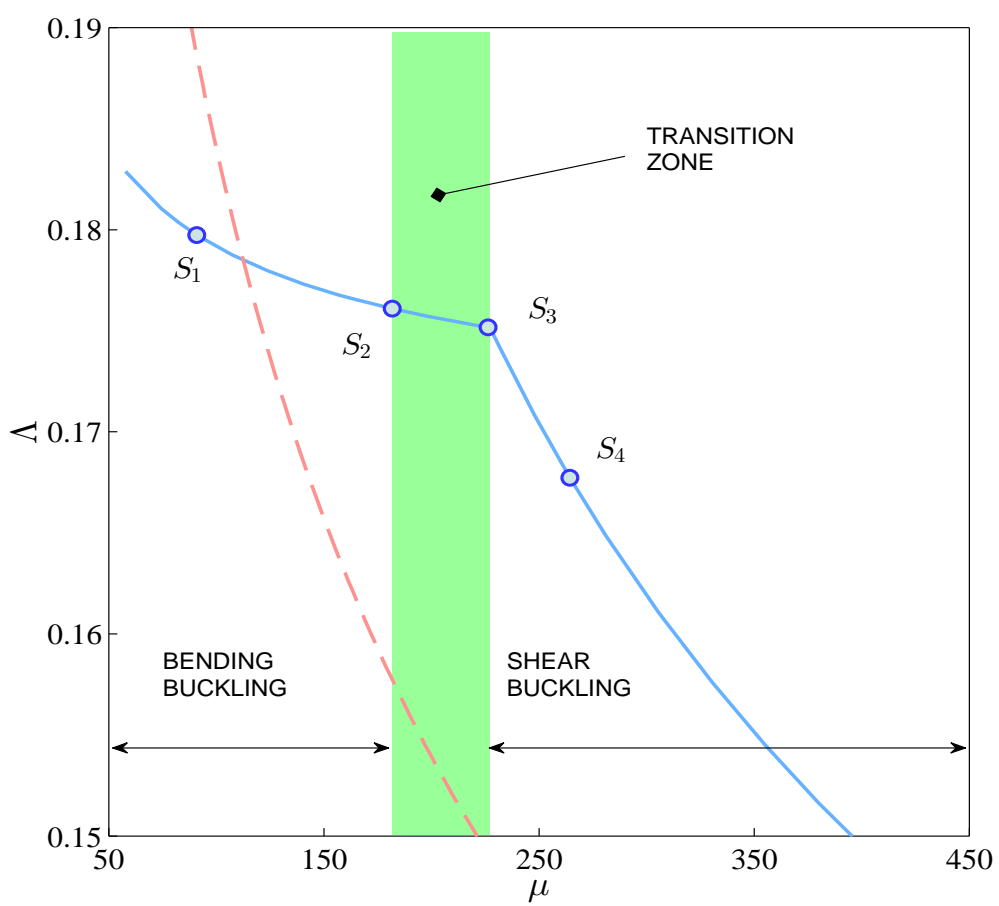

Figure 9: A typical response curve (continuous line) for the boundary-value problem (2.5)(2.6) corresponding to $\alpha=6(\nu=0.3)$. For $R / h \lesssim 110$ the eigen-deformation is localised near the fixed end $(x=1)$, while for $R / h \gtrsim 137.5$ it resembles closely that of a short/mediumlength cylinder subjected to opposite twisting moments around the edges. These regions are bridged by a transition zone (shaded area) in which both modes of deformation co-exist. The dashed line corresponds to Donnell's formula (3.7) and a sample of arbitrary points $S_{j}$ $(j=1,2,3,4)$ have been marked on the curve $\Lambda=\Lambda(\mu)$.

by the cylinder, four arbitrary points $S_{j}(j=1,2,3,4)$ are selected on the $\Lambda$-vs.- $\mu$ curve in Figure 9 and the corresponding eigenmodes are included in Figure 11. Left to the kink, for low values of $\mu$, the deformation of the cylinder is strongly localised near the fixed end, in the vicinity of the point $\left(x_{0}, \theta_{0}\right)$. This localisation persists until $\mu$ has reached the corresponding value for $S_{2}$, which heralds the onset of the growing influence of the shear mode. Between $S_{2}$ and $S_{3}$ the system is in a transitional regime in which shear and bending buckling co-exist, although the latter seems to be the dominant effect. The point $S_{3}$ marks (roughly) the beginning of the shear-buckling regime, in which the deformation is predominantly spread out over most of the surface of the cylinder. By its very nature, the transition zone has an approximate character; we have identified its size by inspecting the numerical solutions and comparing the magnitudes of the various two modes of deformation. According to the additional information included in Figure 10 it seems that the width of this transition strip is 
inversely proportional to some power of $\mu$. This is because as $\alpha$ increases, $\mu_{c}$ increases as well. In both of the foregoing figures the dashed curve corresponds to Donnell's formula (3.7), with the shear buckling load in the cantilever cylinder being about 1.16 larger. Finally, Figures 12 and 13 illustrate the deformation of the cylinder for the shear and bending modes, respectively.
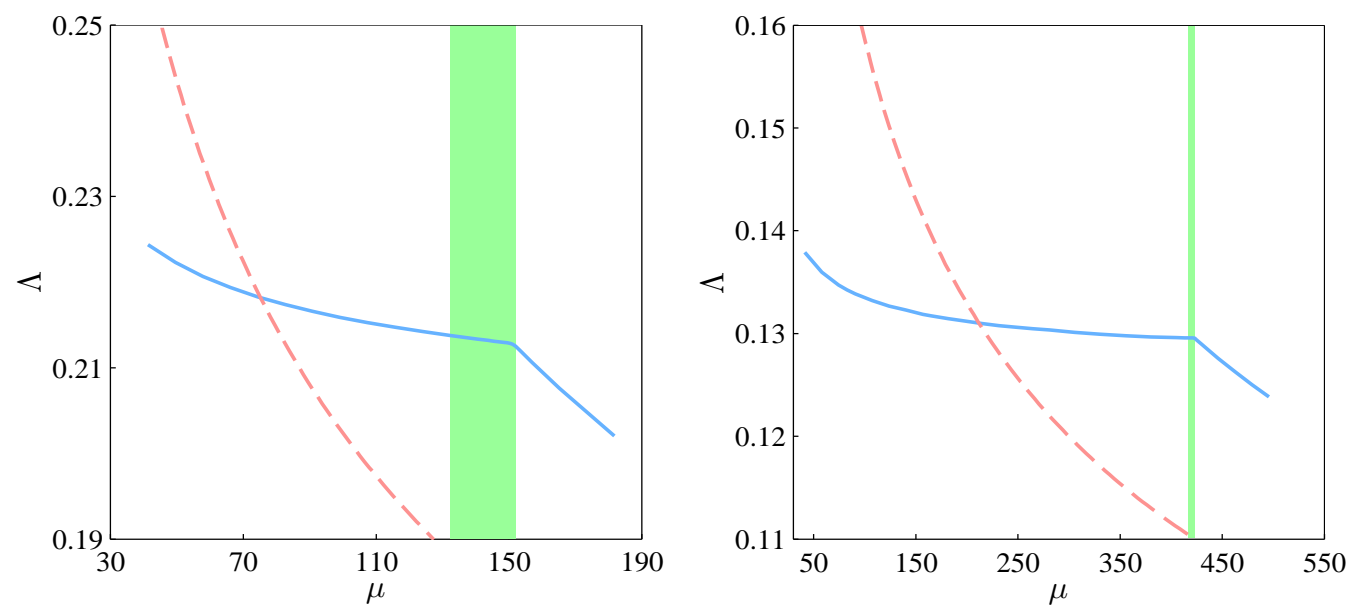

Figure 10: Further examples of response curves for the cantilever cylinder: $\alpha=5$ (left) and $\alpha=8$ (right); in both windows $\nu=0.3$.
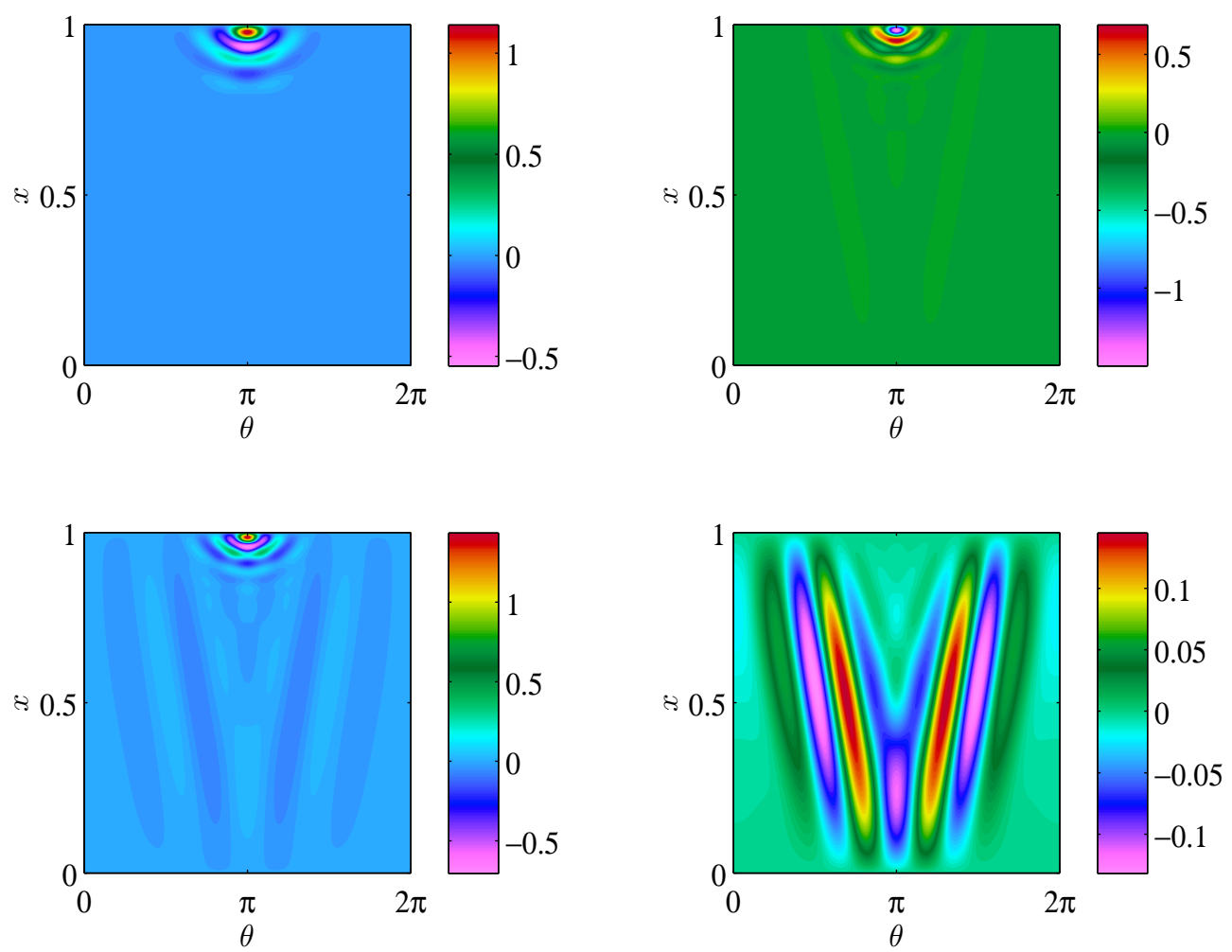

Figure 11: Contour plots illustrating the morphological changes of the eigenmodes corresponding to the round markers recorded on the curve $\Lambda=\Lambda(\mu)$ in Fig. 9: $S_{1}$ (top left), $S_{2}$ (top right), $S_{3}$ (bottom left) and $S_{4}$ (bottom right). Each subplot contains the same number of contour lines. 


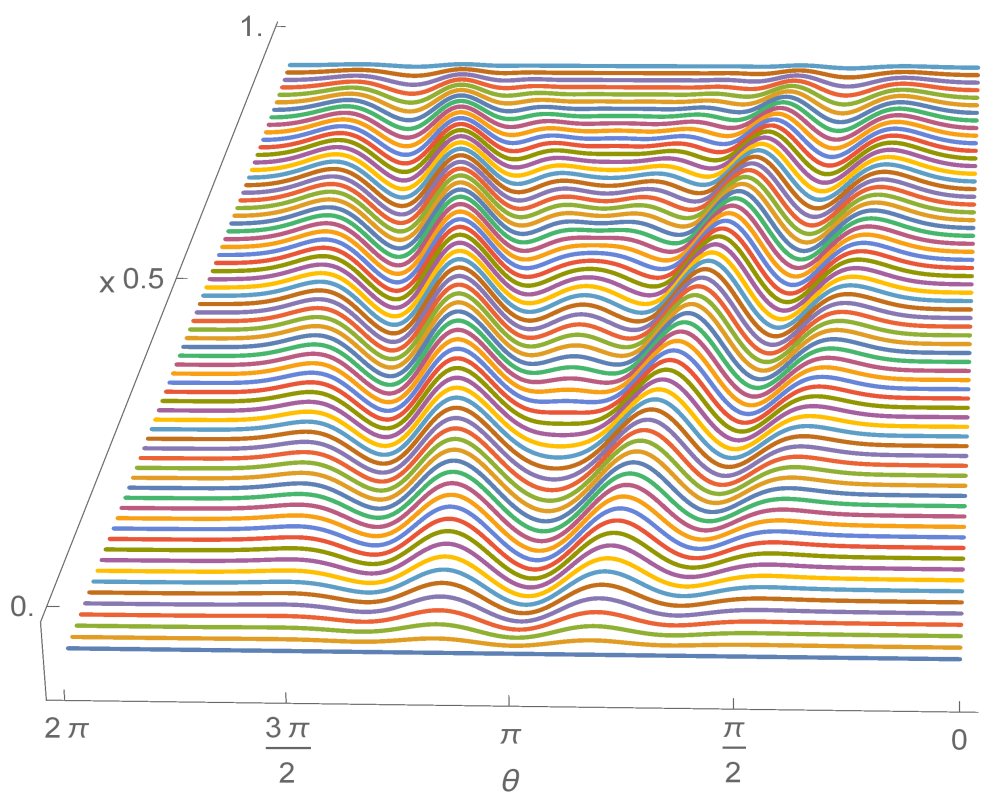

Figure 12: The deformation of a typical shear mode $(\alpha=6, \mu \simeq 30)$. In the interest of clarity the cylinder is developed in the $(x, \theta)$ plane.

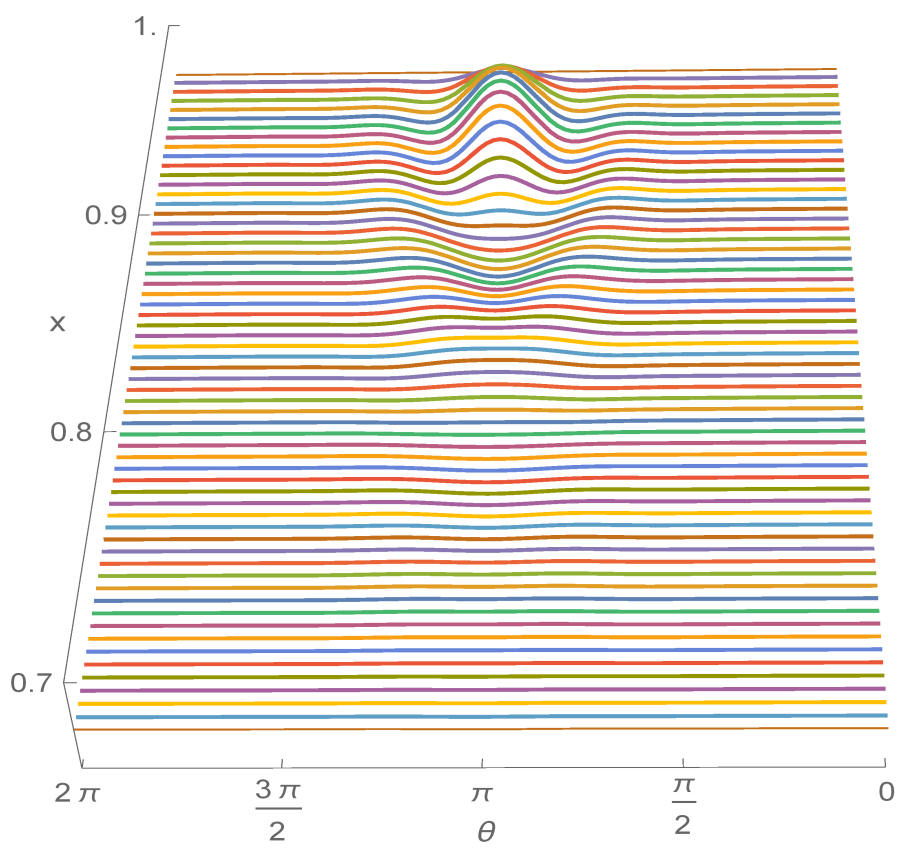

Figure 13: Typical bending mode for $\alpha=6$ and $\mu \simeq 260$; the axial range is restricted to $0.7 \leq x \leq 1$, in order to enhance the details of the localised eigen-deformation.

\section{$5 \quad$ An axial approximation}

Since bending buckling is localised both axially and circumferentially, it is of interest to know if these effects can be separated, at least approximately. In this section we pursue an axial approximation, in 
the sense that we neglect what happens in the circumferential direction; this is based on the observation that the numerical solution varies on a slower spatial scale in the latter direction. The situation we have in mind is idealised in Figure 14 and concerns the behaviour of an infinitesimally narrow strip situated at the top of the cylinder $\left(0 \leq x \leq 1\right.$ and $\left.\theta \simeq \theta_{0} \equiv \pi\right)$.

With this in mind the original equations (2.5) are reduced to

$$
\begin{aligned}
& \frac{\partial^{4} w}{\partial x^{4}}-\alpha^{2} \frac{\partial^{2} F}{\partial x^{2}}+4 \mu \alpha^{3} \Lambda x \frac{\partial^{2} w}{\partial x^{2}}=0, \\
& \frac{\partial^{4} F}{\partial x^{4}}+4 \mu^{2} \alpha^{2} \frac{\partial^{2} w}{\partial x^{2}}=0 .
\end{aligned}
$$

For consistency with the rest of the paper we still keep the partial differentiation symbol in these equations, although we disregard completely the $\theta$-dependence.

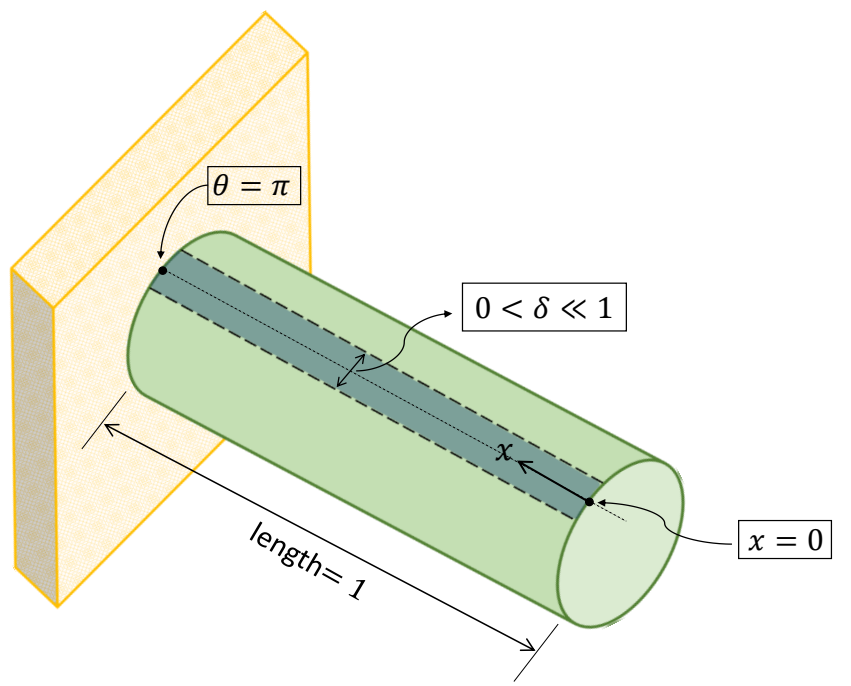

Figure 14: The axial approximation: the imaginary narrow strip whose behaviour is assumed to be described by the system (5.1); the width of the strip is $0<\delta \ll 1$ (i.e., infinitesimally narrow). Both ends of the cylinder are taken to be simply supported, but in the interest of clarity the fixed end is shown as being attached to a vertical "wall".

To investigate the asymptotic structure of the reduced system (5.1), we start by introducing the re-scaled coordinates

$$
X_{1} \equiv \mu^{1 / 2}\left(x_{0}-x\right), \quad X_{2} \equiv \mu^{1 / 3}\left(x_{0}-x\right)
$$

and note that the derivatives with respect ' $x$ ' are transformed according to

$$
\frac{\partial}{\partial x}=-\mu^{1 / 2} D_{1}-\mu^{1 / 3} D_{2}, \quad D_{j} \equiv \frac{\partial}{\partial X_{j}} \quad(j=1,2),
$$

generalised appropriately to higher-order derivatives. We look for a solution of (5.1) with an ansatz of the form

$$
\begin{aligned}
& \boldsymbol{u}=\boldsymbol{u}^{(0)}\left(X_{1}, X_{2}\right)+\mu^{-1 / 6} \boldsymbol{u}^{(1)}\left(X_{1}, X_{2}\right)+\mu^{-1 / 3} \boldsymbol{u}^{(2)}\left(X_{1}, X_{2}\right)+\ldots, \\
& \Lambda=\Lambda_{0}+\Lambda_{1} \mu^{-1 / 3}+\Lambda_{2} \mu^{-1 / 2}+\ldots,
\end{aligned}
$$


where

$$
\boldsymbol{u}:=\left[\begin{array}{c}
w \\
\mu^{-1} F
\end{array}\right], \quad \boldsymbol{u}^{(j)}:=\left[\begin{array}{c}
w_{j} \\
F_{j}
\end{array}\right], \quad(j=0,1,2, \ldots) ;
$$

the constants $\Lambda_{j}(j=0,1, \ldots)$ and the individual terms $\boldsymbol{u}^{(j)}$ on the right-hand side of $(5.4 \mathrm{a})$ are found as usual by substituting the assumed solution in the differential equations, collecting like-powers of $\mu$, and setting to zero the corresponding coefficients. By following this route, at leading order we find

$$
\left[\begin{array}{cc}
D_{1}^{2}\left(D_{1}^{2}+4 \alpha^{3} \Lambda_{0}\right) & -\alpha^{2} D_{1}^{2} \\
4 \alpha^{2} D_{1}^{2} & D_{1}^{4}
\end{array}\right]\left[\begin{array}{c}
w_{0} \\
F_{0}
\end{array}\right]=\left[\begin{array}{l}
0 \\
0
\end{array}\right] .
$$

Since this is a constant-coefficient system we look for solutions in the form $w_{0}=\widehat{w}_{0} \exp \left(p X_{1}\right)$ and $F_{0}=\widehat{F}_{0} \exp \left(p X_{1}\right)$, where $p, \widehat{w}_{0}$ and $\widehat{F}_{0}$ are yet to be found. The characteristic equation satisfied by $p \in \mathbb{C}$ can be re-arranged to give an expression for $\Lambda_{0}=\Lambda_{0}(p)$, namely

$$
\Lambda_{0}=-\frac{1}{4 \alpha^{3}}\left(p^{2}+\frac{4 \alpha^{4}}{p^{2}}\right)
$$

Looking for the minimum positive value of this expression it is found that $p^{2}=-2 \alpha^{2}$, and hence

$$
\Lambda_{0}=\frac{1}{\alpha} .
$$

The solution of (5.5) is then given by

$$
w_{0}=A\left(X_{2}\right) \sin \left(\omega_{0} X_{1}\right), \quad F_{0}=2 w_{0}, \quad \text { where } \quad \omega_{0}:=\alpha \sqrt{2},
$$

while the slowly varying function $A \equiv A\left(X_{2}\right)$ will be fixed at higher orders.

If we let $\boldsymbol{L}$ be the matrix-differential operator that appears on the left-hand side in (5.5), then the next-order equations can be cast in the form

$$
\boldsymbol{L} \boldsymbol{u}^{(1)}=\boldsymbol{f}^{(1)}
$$

where $\boldsymbol{f}^{(1)}:=\left[\mathcal{R}_{11}, \mathcal{R}_{12}\right]^{T}$ (here, ' $T$ ' stands for 'transpose'), with

$$
\mathcal{R}_{11}:=2 \alpha^{2} D_{1} D_{2} F_{0}-4 D_{1}^{3} D_{2} w_{0}-8 \Lambda_{0} \alpha^{3} D_{1} D_{2} w_{0}, \quad \mathcal{R}_{12}:=-8 \alpha^{2} D_{1} D_{2} w_{0}-4 D_{1}^{3} D_{2} F_{0} .
$$

Note that $\mathcal{R}_{12}=2 \mathcal{R}_{11}$, which ensures the consistency of the first-order problem, so the solution of the system (5.9) assumes the form

$$
w_{1}=B\left(X_{2}\right) \cos \left(\omega_{0} X_{1}\right), \quad F_{1}=2 w_{1}+4 \omega_{0}^{-1} A^{\prime}\left(X_{2}\right) \cos \left(\omega_{0} X_{1}\right),
$$

for some $B \equiv B\left(X_{2}\right)$ which is determined at higher orders.

The arbitrary function $A\left(X_{2}\right)$ in (5.8) is fixed by considering the equations at the second-order,

$$
\boldsymbol{L} \boldsymbol{u}^{(2)}=\boldsymbol{f}^{(2)}
$$

where $\boldsymbol{f}^{(2)}:=\left[\mathcal{R}_{21}, \mathcal{R}_{22}\right]^{T}$, with

$$
\begin{aligned}
\mathcal{R}_{21}:=\alpha^{2} D_{2}^{2} F_{0}+2 \alpha^{2} D_{1} D_{2} F_{1} & -6 D_{1}^{2} D_{2}^{2} w_{0}-4 D_{1}^{3} D_{2} w_{1}-4 \Lambda_{0} \alpha^{3} D_{2}^{2} w_{0} \\
& -8 \Lambda_{0} \alpha^{3} D_{1} D_{2} w_{1}+4 \Lambda_{0} \alpha^{3} X_{2} D_{1}^{2} w_{0}-4 \Lambda_{1} \alpha^{3} D_{1}^{2} w_{0},
\end{aligned}
$$


and

$$
\mathcal{R}_{22}:=-6 D_{1}^{2} D_{2}^{2} F_{0}-4 D_{1}^{3} D_{2} F_{1}-4 \alpha^{2} D_{2}^{2} w_{0}-8 \alpha^{2} D_{1} D_{2} w_{1} .
$$

The consistency of (5.11) requires that

$$
\mathcal{L}^{\#}[A] \equiv A^{\prime \prime}-\alpha^{2}\left(X_{2}-\alpha \Lambda_{1}\right) A=0,
$$

where the dash denotes differentiation with respect to $X_{2}$. This is easily recognised as being just a re-scaled version of the Airy equation that can be easily put into the standard form by introducing $Y:=\alpha^{2 / 3}\left(X_{2}-\alpha \Lambda_{1}\right)$, whereupon it follows that $d^{2} \bar{A} / d Y^{2}-Y \bar{A}=0$, with $\bar{A}(Y) \equiv A\left(X_{2}\right)$. To fix $\Lambda_{1}$ we need to recall the original boundary conditions (2.6). Since $x=1$ corresponds to $X_{1}=0$, the first boundary condition at the fixed end of the cylinder will be satisfied because of (5.8). To ensure that the second constraint in (2.6) is also taken care of, by using (5.3) we discover the additional requirement that $A^{\prime}\left(X_{2}\right)=0$ for $X_{2}=0$, which eventually leads to

$$
\Lambda_{1}=\frac{\zeta_{0}}{\alpha^{5 / 3}}, \quad \text { with } \quad \zeta_{0} \simeq 1.01879 ;
$$

the value $\left(-\zeta_{0}\right)$ is the first zero of the derivative of the Airy function, i.e. $\operatorname{Ai}^{\prime}\left(-\zeta_{0}\right)=0$.

To summarise, we have found a two-term approximation for the system (5.1) in the form

$$
\Lambda \simeq \Lambda_{\mathrm{ax}}:=\frac{1}{\alpha}+\left(\frac{\zeta_{0}}{\alpha^{5 / 3}}\right) \mu^{-1 / 3}+\ldots .
$$

At this stage it is necessary to evaluate the performance of the approximation obtained above vis-á-vis both the reduced system and the full boundary-value problem (2.5)-(2.6). The corresponding results are included in Figure 15. Although the dependence of $\Lambda$ on $\mu$, as predicted by the simplified axial model, has a slower rate of decay than that of the original bifurcation problem, the two-term asymptotic formula derived above $\left(\Lambda_{\mathrm{ax}}\right)$ remains below the full numerical result up to the transition point. In the case $\alpha=6$, seen in the left window of Figure 15, the range of $\mu$-values corresponding to bending buckling is relatively small $(R / h \lesssim 137.5)$ and the predictions of the simplified model overtake those of the bifurcation system (2.5)-(2.6) around $R / h \simeq 103$ (i.e., $\mu \simeq 170$ ). Since the transition zone showed in Figure 9 sets in at $R / h \simeq 110$ this is not entirely surprising. By contrast, due to the fact that the transition region is much narrower in the case $\alpha=8$ (cf. Figure 10) and the switching between the two distinct types of buckling occurs at larger $\mu$-values, the comparisons between the reduced axial model and the full numerical solution improve drastically - see the right window in the same figure. The localisation of the axial deformation predicted by (5.1) is illustrated in Figure 16 for $R / h=100$ (left window) and $R / h=300$ (right window). The leading-order asymptotic form given by (5.8) and (5.14) represents a fairly accurate approximation, although we remark in passing that the modulation of the sine spatial oscillation is somewhat slower than its counterpart observed in the direct numerical simulations of the original bifurcation problem. 

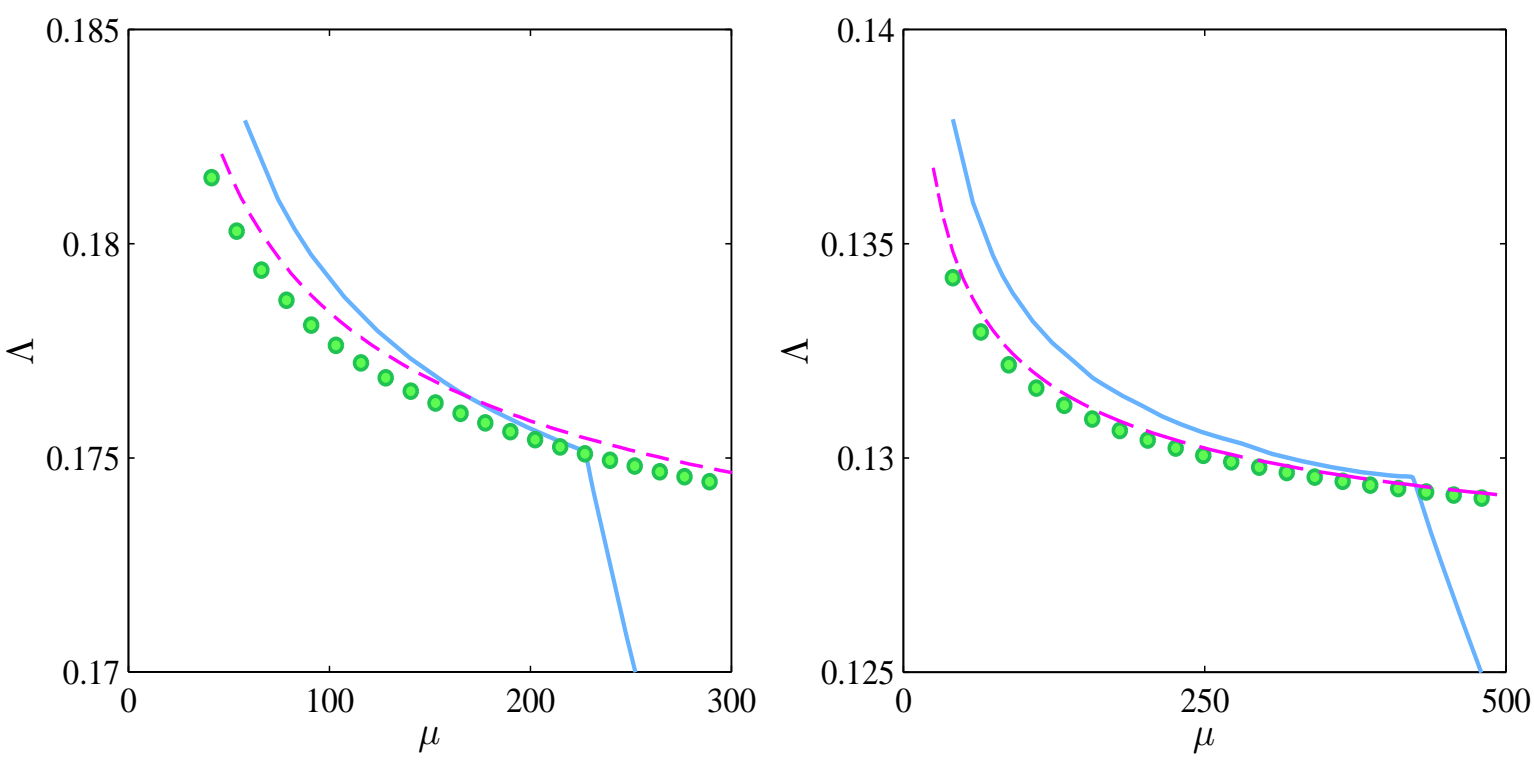

Figure 15: Comparisons of the asymptotic formula (5.16) (discrete markers) with the reduced axial model (5.1) (dashed line) and the full numerical solution of (2.5)-(2.6) (continuous line). Here, $\alpha=6$ (left) and $\alpha=8$ (right).
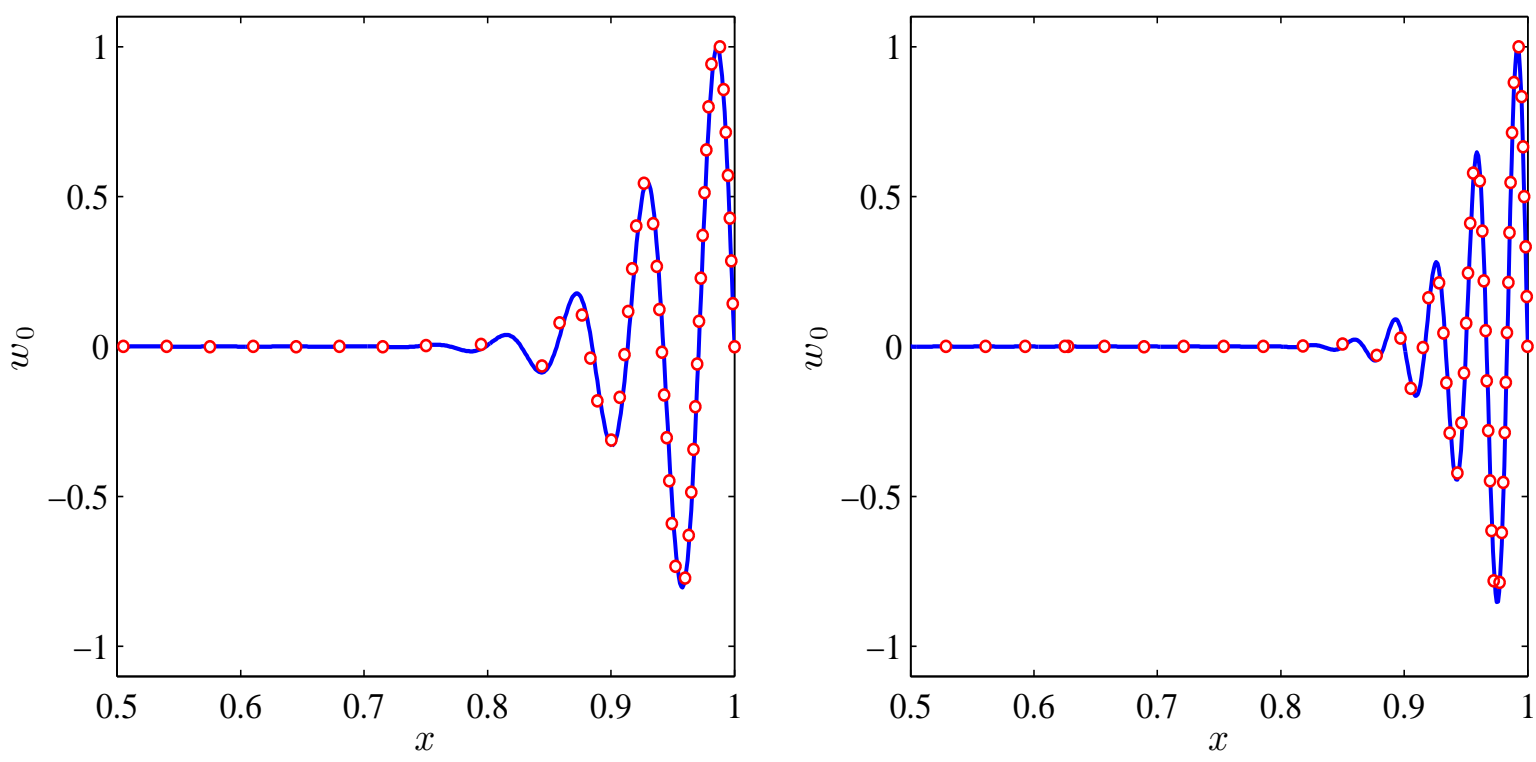

Figure 16: Eigenmodal approximations: the leading-order asymptotic term (5.4a) (continuous line) and the full numerical solution of (5.1) (discrete markers). Here, $R / h=100$ in the left window, and $R / h=300$ on the right; in both sets of plots $\nu=0.3$ and the axial range has been restricted to $1 / 2 \leq x \leq 1$ in the interest of clarity.

\section{The azimuthal localisation}

In this section we use the reduced axial model of $\S 5$ to derive another simplified system of bifurcation equations meant to approximate the localised circumferential deformation of the cantilever cylinder see Figure 17 for a sketch of the situation we have in mind. To this end it will be assumed that the 


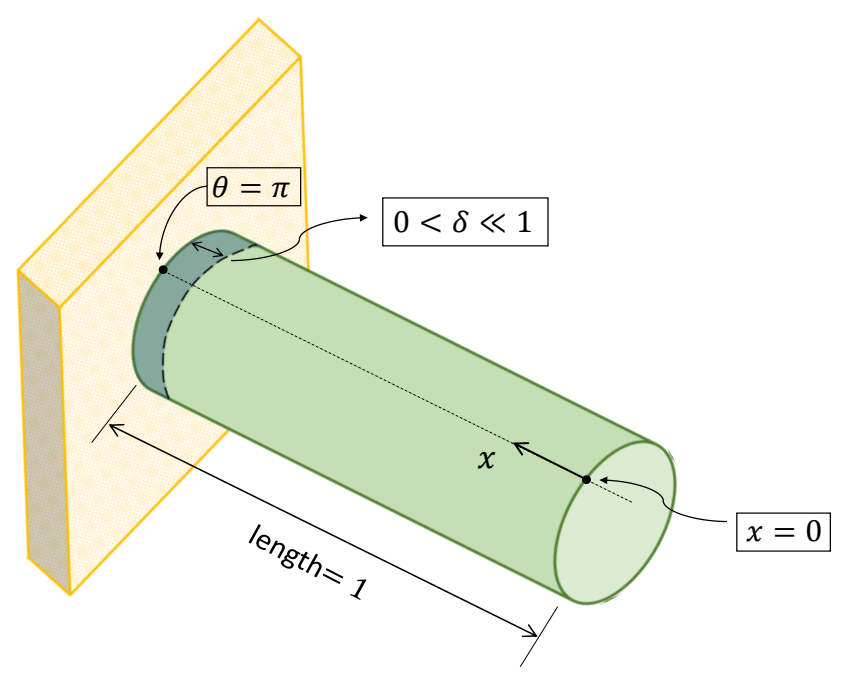

Figure 17: The circumferential approximation: the imaginary terminal ring whose behaviour is described by the system (17).

solution of (2.5) can be expressed in the form

$$
w(x, \theta)=w_{0}(x) G(\theta)+\ldots \quad \text { and } \quad F(x, \theta)=F_{0}(x) H(\theta)+\ldots,
$$

where the 'dots' stand for higher-order terms (in which the ' $x$ ' and ' $\theta$ ' variables might not be separated).

To use the above ansatz in the original partial differential system (2.5) the functions (6.1) need to be differentiated several times with respect to ' $x$ '; we do this by keeping only the dominant behaviour in the axial direction. The final result turns out to be a couple of ordinary differential equations for $G \equiv G(\theta)$ and $H \equiv H(\theta)$ whose coefficients are multiplied by $w_{0}(x)$ or $\cot \left(\omega_{0} X_{1}\right) w_{0}(x)$. In the equation that follows from $(2.5 \mathrm{~b})$ the $x$-dependence can be trivially eliminated, but that is not the case with (2.5a). To accomplish this, the resulting equation is integrated over the length of the cylinder, and we are thus led to introduce the auxiliary quantities

$$
\begin{gathered}
\mathcal{J}_{0}:=\int_{0}^{1} x \operatorname{Ai}\left(\alpha^{2 / 3} X_{2}-\zeta_{0}\right) \sin \left(\omega_{0} X_{1}\right) \mathrm{d} x \\
\mathcal{J}_{1}:=\int_{0}^{1} \operatorname{Ai}\left(\alpha^{2 / 3} X_{2}-\zeta_{0}\right) \cos \left(\omega_{0} X_{1}\right) \mathrm{d} x, \quad \mathcal{J}_{2}:=\int_{0}^{1} \operatorname{Ai}\left(\alpha^{2 / 3} X_{2}-\zeta_{0}\right) \sin \left(\omega_{0} X_{1}\right) \mathrm{d} x .
\end{gathered}
$$

The asymptotic behaviour of these integrals as $\mu \gg 1$ is discussed in Appendix A; in particular, it transpires that $\mathcal{J}_{0} / \mathcal{J}_{2}=\Gamma_{0}+\ldots$ and $\mathcal{J}_{1} / \mathcal{J}_{2}=\Gamma_{1} \mu^{-1 / 2}+\ldots$, where $\Gamma_{j}=\mathcal{O}(1)(j=0,1)$ are known and the dots stand for omitted terms that do no affect the calculations included below. The final (simplified) equations that approximate the azimuthal behaviour of the bifurcation system (2.5) can now be stated in the form

$$
\begin{aligned}
& \frac{\partial^{4} G}{\partial \theta^{4}}-4 \mu \frac{\partial^{2} G}{\partial \theta^{2}}-8 \sqrt{2} \mu \Lambda \Gamma_{1} \sin \theta \frac{\partial G}{\partial \theta}+4 \mu^{2}\left(1+2 \alpha \Lambda \Gamma_{0} \cos \theta\right) G+4 \mu^{2} H=0, \\
& \frac{\partial^{4} H}{\partial \theta^{4}}-4 \mu \frac{\partial^{2} H}{\partial \theta^{2}}+4 \mu^{2} H-4 \mu^{2} G=0 .
\end{aligned}
$$

The new system (6.3) must be solved subject to appropriate periodicity conditions for $G(\theta)$ and $H(\theta)$ on $[0,2 \pi]$, and it represents a boundary-eigenvalue problem for $\Lambda>0$. As we shall see shortly 
the resulting approximations for this parameter provide a tight and consistent lower bound for the full numerical solutions of the original bifurcation problem (2.5)-(2.6). In the meantime, we can take advantage of the presence of $\mu \gg 1$ in (6.3) to find the basic asymptotic structure of these latter equations.

We start by tentatively introducing the re-scaled coordinates

$$
Y_{1} \equiv \mu^{1 / 2}\left(\theta_{0}-\theta\right), \quad Y_{2} \equiv \mu^{1 / 3}\left(\theta_{0}-\theta\right)
$$

and note that the derivatives with respect ' $\theta$ ' are transformed according to

$$
\frac{\partial}{\partial \theta}=-\mu^{1 / 2} D_{1}-\mu^{1 / 3} D_{2}, \quad D_{j} \equiv \frac{\partial}{\partial Y_{j}} \quad(j=1,2),
$$

generalised appropriately to higher-order derivatives. To avoid the proliferation of unnecessary new notations we have denoted the partial differentiation with respect to $Y_{j}$ by $D_{j}(j=1,2)$, as in (5.3). Since the new circumferential scales (6.4) are independent of those in $\S 5$, no confusion should arise.

We look for a solution of the reduced system (6.3) with an ansatz of the form

$$
\begin{aligned}
& \boldsymbol{v}=\boldsymbol{v}^{(0)}\left(Y_{1}, Y_{2}\right)+\mu^{-1 / 3} \boldsymbol{v}^{(1)}\left(Y_{1}, Y_{2}\right)+\mu^{-2 / 3} \boldsymbol{v}^{(2)}\left(Y_{1}, Y_{2}\right)+\mu^{-1} \boldsymbol{v}^{(3)}\left(Y_{1}, Y_{2}\right)+\ldots, \\
& \Lambda=\widehat{\Lambda}_{0}+\widehat{\Lambda}_{1} \mu^{-2 / 3}+\widehat{\Lambda}_{2} \mu^{-4 / 3}+\ldots,
\end{aligned}
$$

where

$$
\boldsymbol{v}:=\left[\begin{array}{c}
G \\
H
\end{array}\right], \quad \boldsymbol{v}^{(j)}:=\left[\begin{array}{c}
G_{j} \\
H_{j}
\end{array}\right], \quad(j=0,1,2, \ldots) ;
$$

the constants $\widehat{\Lambda}_{j}(j=0,1, \ldots)$ and the individual terms $\boldsymbol{v}^{(j)}$ on the right-hand side of (6.6a) are obtained exactly as explained in $\S 5$. At leading order we find the homogeneous differential system

$$
\left[\begin{array}{cc}
\left(D_{1}^{2}-2\right)^{2}-8 \alpha \widehat{\Lambda}_{0} \Gamma_{0} & 4 \\
-4 & \left(D_{1}^{2}-2\right)^{2}
\end{array}\right]\left[\begin{array}{l}
G_{0} \\
H_{0}
\end{array}\right]=\left[\begin{array}{l}
0 \\
0
\end{array}\right] .
$$

Looking for solutions of the form $G_{0}=\widehat{G}_{0} \exp \left(q Y_{1}\right)$ and $H_{0}=\widehat{H}_{0} \exp \left(q Y_{1}\right)$, for some $\widehat{G}_{0}$ and $\widehat{H}_{0}$, results in a linear system for these latter quantities. Its consistency demands that

$$
\widehat{\Lambda}_{0}=\frac{1}{8 \alpha \Gamma_{0}}\left[\left(q^{2}-2\right)^{2}+\frac{16}{\left(q^{2}-2\right)^{2}}\right]
$$

and then minimising this expression with respect to $q \in \mathbb{C}$ gives $q=0$. Thus,

$$
\widehat{\Lambda}_{0}=\frac{1}{\alpha}
$$

where we have used $\Gamma_{0}=1$ (see Appendix A). This also means that the solutions of $(6.3)$ do not depend on $Y_{1}$ and $G_{0}=H_{0}$. Having suppressed the dependence on $Y_{1}$, it remains that $G_{j}=G_{j}\left(Y_{2}\right)$ and $H_{j}=H_{j}\left(X_{2}\right)$ for $j=0,1, \ldots$

Continuing with the asymptotic analysis of $(2.5)$ we note that in the differential operator that appears in (6.7) the derivative plays no role now, and is therefore reduced to an ordinary matrix, $\boldsymbol{M}$ (say). The first inhomogeneous equations obtained can be expressed as $\boldsymbol{M} \boldsymbol{v}^{(1)}=\boldsymbol{f}^{(1)}$, where $\boldsymbol{f}^{(1)}:=\left[\mathcal{R}_{11}, \mathcal{R}_{12}\right]^{T}$, with $\mathcal{R}_{11}=\mathcal{R}_{12}:=-4 D_{2}^{2} G_{0}$; this gives $H_{1}=G_{1}+D_{2}^{2} G_{0}$.

The next in the hierarchy of order equations is $\boldsymbol{M} \boldsymbol{v}^{(2)}=\boldsymbol{f}^{(2)}$, where $\boldsymbol{f}^{(2)}:=\left[\mathcal{R}_{21}, \mathcal{R}_{22}\right]^{T}$, with

$$
\mathcal{R}_{21}:=-D_{2}^{4} G_{0}+4 D_{2}^{2} G_{1}-4 Y_{2}^{2} G_{0}+8 \alpha \widehat{\Lambda}_{1} G_{0} \quad \text { and } \quad \mathcal{R}_{22}:=-D_{2}^{4} H_{0}+4 D_{2}^{2} H_{1} .
$$


The consistency of this system demands that $\mathcal{R}_{21}-\mathcal{R}_{22}=0$, whereby

$$
\mathcal{L}^{\dagger}\left[G_{0}\right] \equiv \frac{d^{4} G_{0}}{d Y_{2}^{4}}+\left(Y_{2}^{2}-2 \alpha \widehat{\Lambda}_{1}\right) G_{0}=0
$$

This equation must be solved subject to the decay conditions at infinity,

$$
G_{0}, \quad \frac{d G_{0}}{d Y_{2}} \rightarrow 0, \quad \text { as } \quad Y_{2} \rightarrow \pm \infty .
$$

Equation (6.9) subject to (6.10) represents a simple boundary-eigenvalue problem that is solved (once and for all) to give

$$
2 \alpha \widehat{\Lambda}_{1}=: \kappa_{1} \simeq 1.060362 .
$$

Given the universal nature of equation (6.9) it is not difficult to calculate the next-order correction in (6.6b). This is done by using the equations $\boldsymbol{M} \boldsymbol{v}^{(3)}=\boldsymbol{f}^{(3)}$, where $\boldsymbol{f}^{(3)}:=\left[\mathcal{R}_{31}, \mathcal{R}_{32}\right]^{T}$, with

$$
\begin{gathered}
\mathcal{R}_{31}:=-D_{2}^{4} G_{1}+4 D_{2}^{2} G_{2}-8 \sqrt{2} \widehat{\Lambda}_{0} \Gamma_{1} Y_{2} D_{2} G_{0}-4 \alpha \widehat{\Lambda}_{0} Y_{2}^{2} G_{1}+8 \alpha \widehat{\Lambda}_{1} G_{1}+8 \alpha \widehat{\Lambda}_{2} G_{0}, \\
\mathcal{R}_{32}:=-D_{2}^{4} H_{1}-4 D_{2}^{2} H_{2} .
\end{gathered}
$$

Subtraction of these two expressions must give zero (to ensure the consistency of the third-order system). Since the second-order equations are equivalent to $-4 G_{2}+4 H_{2}=-D_{2}^{4}+4 D_{2}^{2} H_{1}$, straightforward algebraic manipulations yield an inhomogeneous version of equation (6.9) for $G_{1}=G_{1}\left(Y_{2}\right)$,

$$
\mathcal{L}^{\dagger}\left[G_{1}\right]=-\frac{1}{2} D_{2}^{6} G_{0}-2 \sqrt{2} \widehat{\Lambda}_{0} \Gamma_{1} Y_{2} D_{2} G_{0}+2 \alpha \widehat{\Lambda}_{2} G_{0} .
$$

The application of the usual Fredholm solvability condition in (6.12), followed by repeated integration by parts, finally leads to the desired expression

$$
\widehat{\Lambda}_{2}=\frac{1}{4 \alpha^{3}}\left[2 \kappa_{2}+\alpha^{2}\left(\kappa_{3}+\kappa_{1} \kappa_{4}\right)\right],
$$

where

$$
\kappa_{2}:=\frac{\mathcal{B}_{1111}}{\mathcal{B}_{0002}} \simeq-0.5, \quad \kappa_{3}:=\frac{\mathcal{B}_{2211}}{\mathcal{B}_{0002}} \simeq 0.146450, \quad \kappa_{4}:=\frac{\mathcal{B}_{0220}}{\mathcal{B}_{0002}} \simeq 0.353454,
$$

and for notational convenience we have introduced the following definitions

$$
\mathcal{B}_{i j k l}:=\int_{-\infty}^{+\infty}\left(Y_{2}\right)^{i}\left(D_{2}^{j} G_{0}\right)^{k}\left(G_{0}\right)^{l} \mathrm{~d} Y_{2}, \quad \text { for } \quad i, j, k, l \geq 0 .
$$

In Figure 18 the performance of these analytical formulae is compared with the direct numerical results of the original eigenproblem (2.5)-(2.6) together with its simplified counterpart described by (6.3). As before in $\S 5$ the full numerical solutions are denoted by the continuous (blue) curve, while the predictions using the asymptotic results (6.6b), (6.8), (6.11) and (6.13) are showed as discrete markers; the numerical solutions of the reduced model are represented by the dashed (magenta) curve. Note that here we plot the dependence of the maximum bending stress $(\alpha \Lambda)$ on $\mu$. Although, as expected, the reduced model underestimates the critical loads of the original problem, the relative errors range from $4.4 \%$ on the left $(\alpha=6)$ to $3.9 \%$ in the other window $(\alpha=8)$. In light of the relatively low values of $\mu$ these predictions are reasonable and can be regarded as a reliable lower bound for the true maximum bending stress in the cantilever cylinder. Included in these plots is also a straight line which 

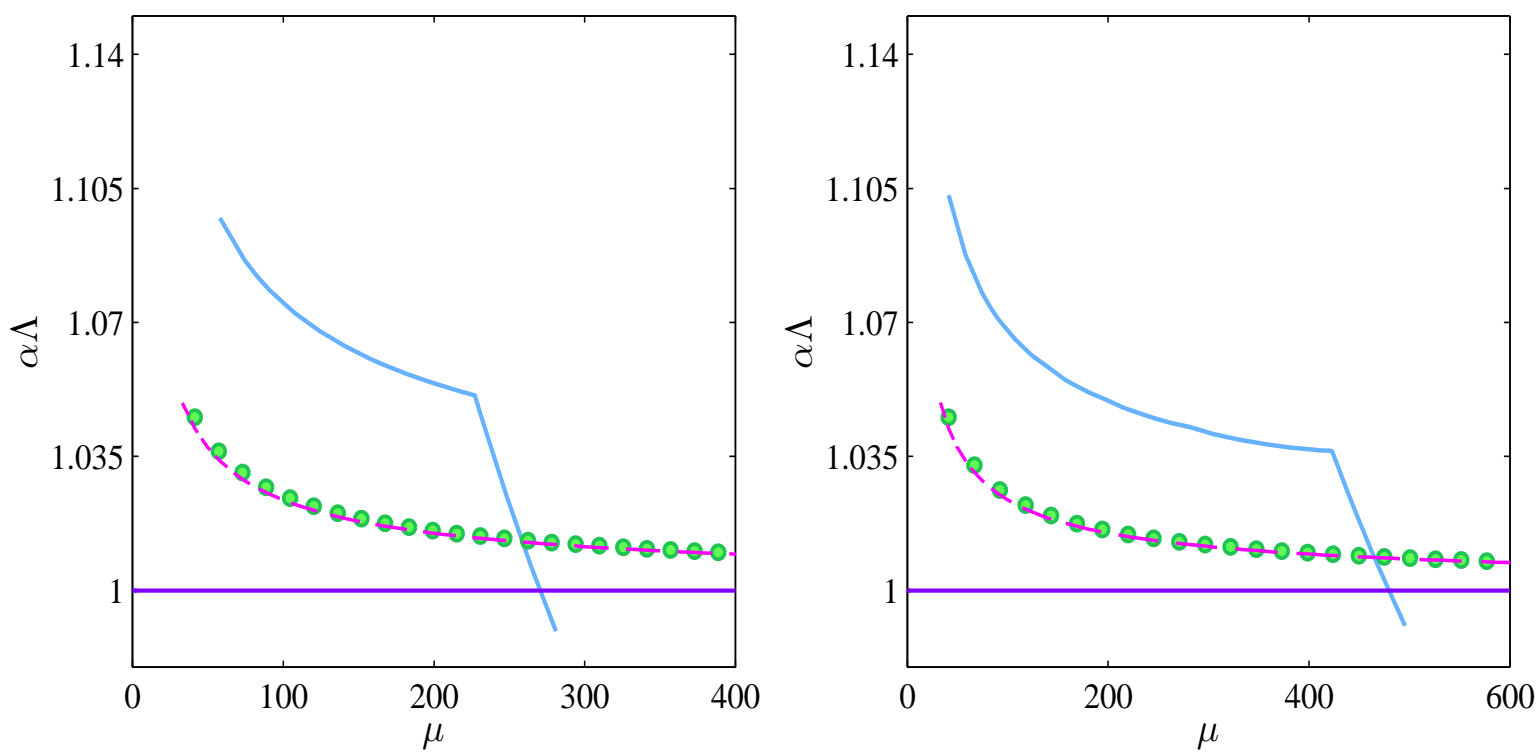

Figure 18: Sample of comparisons for the azimuthal approximation (6.6b): $\alpha=6$ (left) and $\alpha=8$ (right). The asymptotic results correspond to the discrete markers, the numerical solution of the reduced azimuthal system (6.3) is represented by the dashed line, and the full numerical solution of (2.5)-(2.6) is indicated by the continuous curve. Shown in both windows is also the line $\alpha \Lambda=1$ corresponding to the critical load for the buckling of an axially compressed long cylindrical shell.

corresponds to the critical compressive stress for an axially compressed long cylinder (the quantity $\sigma_{*}$ -see the definitions (2.4)).

The solution of the reduced model, as well as its asymptotic approximation, share the feature noticed by Seide \& Weingarten [22], namely, that the maximum bending stresses responsible for buckling in pure bending of short cylinders asymptote to $\sigma_{*}$ as $h \rightarrow 0$. However, this does not seem to be the case for the cantilever cylinder due to the transition point which places an upper bound on how big $\mu$ can be for a given $\alpha$. To appreciate better the nature of this second situation we have plotted in Figure 19 all three sets of direct numerical solutions for (2.5)-(2.6) in terms of $\delta \equiv \mu^{1 / 2} / \alpha$ (see the discussion in $\S 3$ ). Their transition points $T P_{j}(j=5,6,8)$, indicated by the discrete markers, are situated on a curve that intercepts the horizontal line $(\alpha \Lambda=1)$ in a point $T P_{\infty}$ whose abscissa is $\ell_{*} \simeq 2.74034$; also, the shear-buckling parts of the response curves overlap and collapse onto the same curve. While only a limited amount of numerical data is presented in Figure 19, it is easy to anticipate what would happen if more response curves were added. Assuming $\left\{\alpha_{n}\right\}$ to be a set of increasing positive real numbers with $\lim _{n \rightarrow \infty} \alpha_{n}=+\infty$, then the response curve for $\alpha_{n}$ will be situated between those corresponding to $\alpha_{n-1}$ (above) and $\alpha_{n+1}$ (below). Furthermore, all these curves will lie above the straight line $\alpha \Lambda=1$ and their transition points will form a decreasing sequence on the curve indicated in the figure, which tends to the green marker as $n \rightarrow \infty$. This means that the line $\alpha \Lambda=1$ is reached only in the limit $\alpha \rightarrow \infty$ and, since $\mu^{1 / 2} / \alpha \rightarrow \ell_{*}<\infty$ in that limit, we have reasons to suspect that $\mu \rightarrow \infty$ as well. To put it differently, unlike in the pure bending problem recently studied in [23], here the limits $\alpha \rightarrow \infty$ and $\mu \rightarrow \infty$ are interdependent, so the analogy between the current problem and that in the reference just cited is incomplete. We do not speculate further on what happens for $\alpha>8$ because the ovalisation experienced by the cantilever cylinder can no longer be neglected within that regime, and this will require the introduction of nonlinear effects in the basic state.

The numerical information contained in Figure 19 suggests an asymptotic simplification of the 


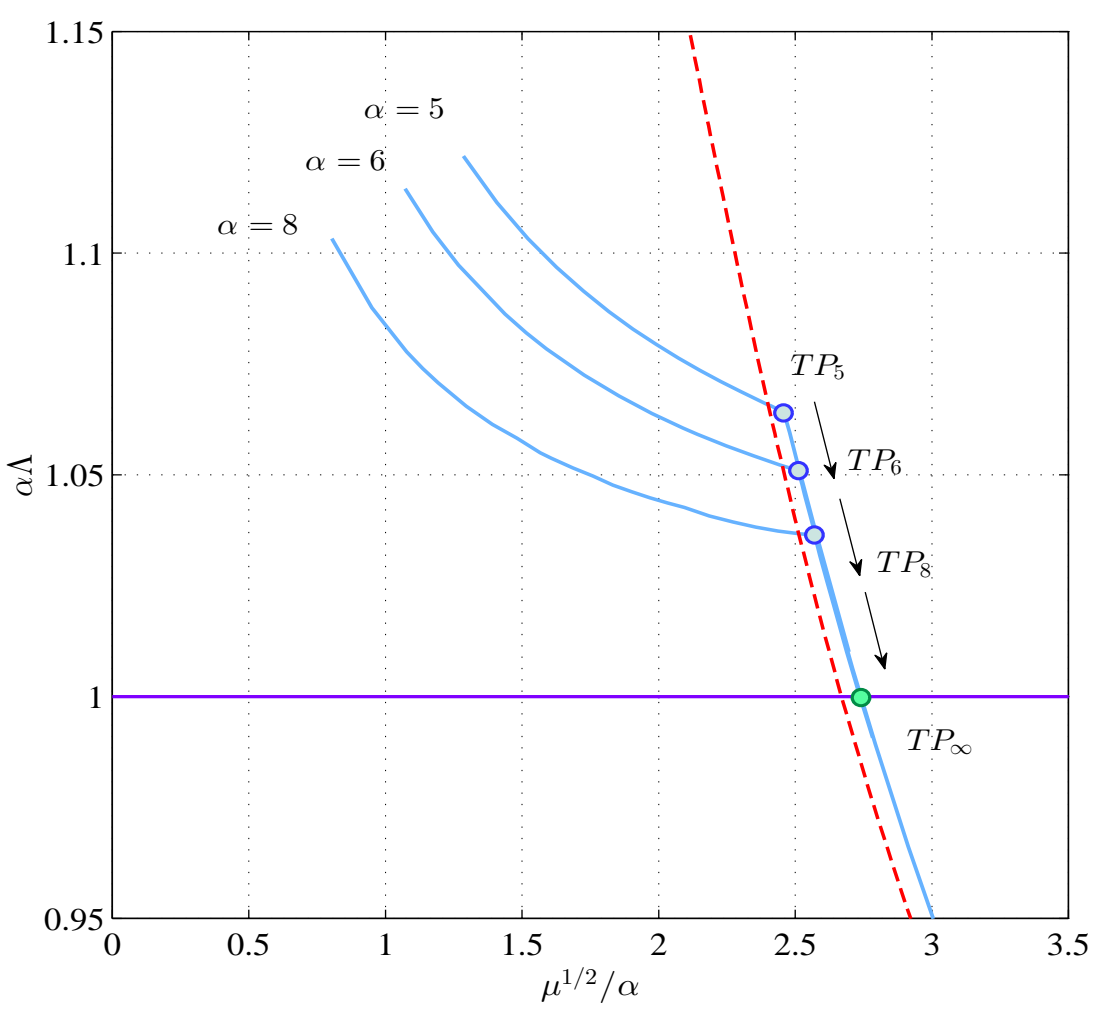

Figure 19: Numerical evidence suggesting that the critical bending buckling stress in the cantilever cylinder approaches $\sigma_{*}$ (see (2.4)) only when both $\alpha \rightarrow \infty$ and $\mu \rightarrow \infty$. Note, $\mu^{1 / 2} / \alpha \rightarrow \ell_{*} \simeq 2.74034$ in that limit - this numerical value corresponds to the horizontal coordinate of the point labelled $T P_{\infty}$. The continuous curves are the same as those included in Figs. 9 and 10 (for clarity their transition points are indicated by round markers), while the dashed curve represents the shear-buckling approximation obtained from the equation (6.16).

original equations that captures the shear-buckling behaviour. To this end, we write $\alpha^{2}=\alpha_{0} \mu+\ldots$, $\Lambda_{*} \equiv \alpha \Lambda=K_{0} \mu^{-1 / 4} \alpha^{1 / 2}$, where $K_{0}>0$ is to be found and $\alpha_{0}>0$ is taken as given. By further letting $w(x, \theta)=w_{0}(x, \theta)+\ldots, F(x, \theta)=\mu F_{0}(x, \theta)+\ldots$, and then performing the corresponding substitutions in $(2.5)$, gives

$$
\begin{aligned}
& \alpha_{0} \frac{\partial^{4} w_{0}}{\partial \theta^{4}}-\frac{\partial^{2} F_{0}}{\partial x^{2}}-4 K_{0} \alpha_{0}^{1 / 4}\left[x \cos \theta\left(\frac{\partial^{2} w_{0}}{\partial x^{2}}\right)-2 \sin \theta\left(\frac{\partial^{2} w_{0}}{\partial x \partial \theta}\right)\right]=0, \\
& \alpha_{0} \frac{\partial^{4} F_{0}}{\partial \theta^{4}}+4 \frac{\partial^{2} w_{0}}{\partial x^{2}}=0
\end{aligned}
$$

which must be solved subject to the same boundary conditions as the original full system - see (2.6). In deriving the above form of these equations we have kept only the dominant terms (e.g., the terms omitted in the first equation were of order $\mathcal{O}\left(\mu^{-1}\right)$ and $\mathcal{O}\left(\mu^{-2}\right)$, etc). In Figure 19 the dependence of $K_{0} \alpha_{0}^{1 / 4} \equiv \alpha \Lambda$ on $\alpha_{0}^{-1 / 2} \equiv \mu^{1 / 2} / \alpha$ is shown as the dashed (red) curve. The relative errors between this curve and the full numerical simulations is less than $3 \%$; given that what we have here is the leading-order of a regular perturbation problem, and that shear-buckling involves a diffuse deformation pattern, this agreement is actually quite encouraging. 


\section{Concluding remarks}

We have investigated the linear elastic instabilities of a thin cantilever cylindrical shell subjected to a vertical shear force at its free end. The emphasis here has been on a localised form of buckling that bears considerable resemblance to the corresponding eigenmodal deformation of a thin isotropic cylinder loaded in pure bending [22, 23, 24]. Motivated by the doubly-localised nature of the eigenmodes in the former case, we have been mainly interested in exploring an approximate asymptotic separation of the axial and circumferential coordinates, as well as the repercussions this has on the analytical approximation of the critical buckling loads. By first ignoring the dependence on the circumferential coordinate, the corresponding axial system was then found to be amenable to a double-scale asymptotic analysis that provided close predictions to the numerical simulations of the full bifurcation equations (cf. §5). The azimuthal localisation of the buckling patterns was also partially captured by further assuming that the quantities of interest (transverse displacement and stress function) can be expressed as a product between the preceding axial fields and unknown azimuthal functions; the latter were subsequently identified from the solution of a different ordinary differential system with the help of another double-scale asymptotic procedure. Our main results consist of simple asymptotic predictions for the critical shear force $\Lambda$ as a function of the relative thickness and length of the cylinder, $\mu$ and $\alpha$, respectively - for their precise definitions see $(2.3 \mathrm{~b})$; these formulae appear summarised in (5.16) and (6.6b), together with (6.8), (6.11) and (6.13).

At first sight, the route followed in this paper might seem to be reminiscent of Kantorovich's classical approximation method for partial differential equations with non-separable variables [31] (see also the interesting work of Kerr et al. [32, 33, 34]). However, this analogy is only superficial. Although in $\S 6$ the $x$-dependence was indeed prescribed right from the outset, this was not done in an arbitrary way by simply satisfying just the kinematic boundary conditions. Another key difference lies in the fact that the $\theta$-dependence in our case was obtained from the original bifurcation equations after taking an average in the $x$-direction, rather than using the variational form of the problem. It is quite likely that the Kantorovich method will not perform well on this problem due to the presence of multiple spatial scales in the eigen-deformation pattern (unless, of course, one makes some very special choices for the $x$ - or $\theta$-dependence).

The membrane basic state adopted in this work does impose some limitations, and for this reason we have restricted the range of $\alpha$-values to those for which the nonlinear effects in the pre-buckling stage can be safely regarded as negligible (cf. [9]). For truly long cylinders (i.e., $\alpha \geq 10$ ) the ovalisation of the cross-section does start to play an increasingly important role and has the effect of shifting the localised buckling pattern away from the fixed end, although it remains confined in that region. This is corroborated by experiments as it can be seen, for instance, in Figure 2(b,c) of reference [11]. The same conclusions are also delivered by the simple-minded model proposed by Hangai et al. [16] for a cylinder with the same boundary conditions as in present work. It is thus of interest to extend the analysis presented herein for the case of a nonlinear pre-buckling state, and we hope to report the corresponding results in due course.

\section{A Asymptotic behaviour of the integrals (6.2)}

We sketch below the asymptotic behaviour of the integrals that appear in $\S 6$. Note that we can write

$$
\mathcal{J}_{1}+\mathrm{i} \mathcal{J}_{2}=\int_{0}^{1} \psi(x) \exp \left[\mathrm{i} \mu^{1 / 2} \omega_{0}(1-x)\right] \mathrm{d} x,
$$

with

$$
\psi(x):=\operatorname{Ai}\left(\alpha^{2 / 3} \mu^{1 / 3}(1-x)-\zeta_{0}\right) .
$$


Repeated integration by parts ( $N$ times, for some $N \geq 1$ ) of the right-hand side of (A.1) gives

$$
\mathcal{J}_{1}+\mathrm{i} \mathcal{J}_{2}=\frac{\mathrm{i} \mu^{-1 / 2}}{\omega_{0}}\left\{\sum_{k=0}^{N-1} A_{k}^{(1)} \mu^{-k / 2}\right\}-\frac{\mathrm{ie}^{\mathrm{i} \mu^{1 / 2} \omega_{0}}}{\omega_{0} \mu^{1 / 2}}\left\{\sum_{k=0}^{N-1} A_{k}^{(0)} \mu^{-k / 2}\right\}+\mathcal{O}\left(\mu^{-N / 2}\right),
$$

where $A_{k}^{(s)}:=(-\mathrm{i})^{k} \omega_{0}^{-k} \psi^{(k)}(s)$ for $s=0,1$ and $k=0,1,2, \ldots, N-1$. Due to the asymptotic behaviour of the Airy function 'Ai' at $+\infty$ the second term in (A.2) will be exponentially small, and hence it can be discarded. Evaluating the coefficients in the first term and then separating the real and imaginary parts of the resulting expression it follows that

$$
\begin{aligned}
& \mathcal{J}_{1}=\frac{\mathrm{Ai}_{0}}{\omega_{0}}\left(\mathcal{A}_{1} \mu^{-1}+\mathcal{A}_{2} \mu^{-4 / 3}+\mathcal{A}_{3} \mu^{-5 / 3}+\ldots\right), \\
& \mathcal{J}_{2}=\frac{\mathrm{Ai}_{0}}{\omega_{0}}\left(\mu^{-1 / 2}+\mathcal{B}_{2} \mu^{-5 / 6}+\mathcal{B}_{3} \mu^{-7 / 6}+\mathcal{B}_{4} \mu^{-3 / 2}+\mathcal{B}_{5} \mu^{-11 / 6}+\ldots\right),
\end{aligned}
$$

where $\operatorname{Ai}_{0}:=\operatorname{Ai}\left(-\zeta_{0}\right)$ and

$$
\begin{gathered}
\mathcal{A}_{1}:=\frac{1}{2 \sqrt{2} \alpha}, \quad \mathcal{A}_{2}:=\frac{\zeta_{0}}{\sqrt{2} \alpha^{5 / 3}}, \quad \mathcal{A}_{3}:=\frac{9 \zeta_{0}^{2}}{8 \sqrt{2} \alpha^{7 / 3}}, \\
\mathcal{B}_{2}:=\frac{\zeta_{0}}{2 \alpha^{2 / 3}}, \quad \mathcal{B}_{3}:=\frac{\zeta_{0}^{2}}{4 \alpha^{4 / 3}}, \quad \mathcal{B}_{4}:=\frac{\zeta_{0}^{3}-4}{8 \alpha^{2}}, \quad \mathcal{B}_{5}:=\frac{\zeta_{0}\left(\zeta_{0}^{3}-28\right)}{16 \alpha^{8 / 3}} .
\end{gathered}
$$

It is now a simple matter to use these expansions to find

$$
\frac{\mathcal{J}_{1}}{\mathcal{J}_{2}}=\frac{\mu^{-1 / 2}}{2 \sqrt{2} \alpha}\left[1+\left(\frac{3 \zeta_{0}}{2 \alpha^{2 / 3}}\right) \mu^{-1 / 3}+\left(\frac{5 \zeta_{0}^{2}}{4 \alpha^{4 / 3}}\right) \mu^{-2 / 3}+\left(\frac{4-\zeta_{0}^{3}}{8 \alpha}\right) \mu^{-1}\right]+\mathcal{O}\left(\mu^{-11 / 6}\right) .
$$

Regarding the integral $\mathcal{J}_{0}$ defined in $(6.2 \mathrm{a})$, its asymptotic evaluation can also be obtained from (A.1) after making some obvious changes (e.g., $\psi(x) \rightarrow x \psi(x)$, etc). Without going into details, it is found that

$$
\mathcal{J}_{0}=\mu^{-1 / 2}\left(1+\mathcal{C}_{2} \mu^{-1 / 3}+\mathcal{C}_{3} \mu^{-2 / 3}+\mathcal{C}_{4} \mu^{-1}+\mathcal{C}_{5} \mu^{-4 / 3}+\ldots\right) \frac{\mathrm{Ai}_{0}}{\omega_{0}}
$$

where

$$
\mathcal{C}_{2}:=\frac{\zeta_{0}}{2 \alpha^{2 / 3}}, \quad \mathcal{C}_{3}:=\frac{\zeta_{0}^{2}}{4 \alpha^{4 / 3}}, \quad \mathcal{C}_{4}:=\frac{\zeta_{0}^{3}-12}{8 \alpha^{2}}, \quad \mathcal{C}_{5}:=\frac{\zeta_{0}\left(\zeta_{0}^{3}-76\right)}{16 \alpha^{8 / 3}} .
$$

The asymptotic behaviour of the quotient $\mathcal{J}_{0} / \mathcal{J}_{2}$ in the limit $\mu \gg 1$ is described by

$$
\frac{\mathcal{J}_{0}}{\mathcal{J}_{2}}=1-\left(\frac{1}{\alpha^{2}}\right) \mu^{-1}-\left(\frac{5 \zeta_{0}}{2 \alpha^{8 / 3}}\right) \mu^{-4 / 3}+\left(\frac{3 \zeta_{0}^{2}}{2 \alpha^{10 / 3}}\right) \mu^{-5 / 3}+\ldots
$$

\section{References}

[1] Yamaki, N. Elastic Stability of Circular Cylindrical Shells. Amsterdam: North-Holland, 1984.

[2] Brush, DO, Almroth, BO. Buckling of Bars, Plates, and Shells. New York: McGraw Hill Inc., 1975.

[3] Timoshenko, SP, Gere, JM. Theory of Elastic Stability. New York: McGraw Hill Inc., 1961.

[4] Lundquist, EE. Strength tests of thin-walled duralumin cylinders in combined transverse shear and bending. NACA-TN-523, Langley Memorial Aeronautical Laboratory, Washington, DC., 1935 . 
[5] Lu, SY. Buckling of cantilever cylindrical shell with a transverse end load. AIAA Journal 1965; 3: $2350-2351$.

[6] Johns, DJ. On the linear buckling of circular cylindrical shells under asymmetric axial compressive stress distributions. The Aeronautical Journal 1966; 70: 1095-1097.

[7] Lee, RL. Buckling of a stiffened cylindrical shell with transverse shear load. AIAA Journal 1969; 7: $768-769$.

[8] Schröder, P. Über die Stabilität der querkraftbelasteten dünnwandigen Kreiszylinderschale. ZAMM 1972; 52: T145-T148.

[9] Jamal, A. Collapse of anisotropic cylindrical shells under combined bending and transverse shear load. PhD Thesis, Delft University of Technology, Faculty of Aerospace Engineering, The Netherlands, 1998.

[10] Manevich, AI, Ponomarenko, EA, Prokopalo, EF. Stability of orthotropic cylindrical shells under bending by a transverse force. Part 1. Theory. Strength of Materials 2013; 45: 73-81.

[11] Manevich, AI, Ponomarenko, EA, Prokopalo, EF. Stability of orthotropic cylindrical shells under bending by a transverse force. Part 2. Experiment. Strength of Materials 2013; 45: 205-209.

[12] Michel, G, Limam, A, Jullien, JF. Buckling of cylindrical shells under static and dynamic shear loading. Eng. Struct. 2000; 22: 535-543.

[13] Kokubo, K, Nagashima, H, Takayanagi, M, Mochizuki, A. Analysis of shear buckling of cylindrical shells. JSME International Journal, Series A 2000; 36: 259-266.

[14] Nagashima, H, Kokubo, K, Takayanagi, M, Saitoh, K, Imaoka, T. Experimental study on the dynamic buckling of cylindrical tanks. JSME International Journal 2000; 30: 737-746.

[15] Yoneda, M, Ohmori, H, Hangai, Y. Buckling failure of cantilever type cylindrical shells under the horizontal load. Bulletin of Earthquake Resistant Structure Research Centre, Institute of Industrial Science, University of Tokyo 1982; 15: 13-21.

[16] Hangai, Y, Choi, HS. Local buckling of cylindrical tanks under the horizontal load. Bulletin of Earthquake Resistant Structure Research Centre, Institute of Industrial Science, University of Tokyo 1984; 17: 3-11.

[17] Brazier, LG. On the flexure of thin cylindrical shells and other thin sections. Proc. Roy. Soc. A 1927; 116: 104-114.

[18] Axelrad, EL. Theory of Flexible Shells. Amsterdam: North-Holland, 1987.

[19] Axelrad, EL. On local buckling of thin shells. Int. J. Non-Linear Mech. 1985; 20: 249-259.

[20] Rammerstorfer, FG, Scharf, K, Fisher, FD. Storage tanks under earthquake loading. Appl. Mech. Rev. 1990; 43: 261-282.

[21] Teng, JG, Rotter, JM (Eds.). Buckling of Thin Metal Shells. London \& New York: Spon Press, 2004.

[22] Seide, P, Weingarten, VI. On the buckling of circular cylindrical shells under pure bending. ASME J. Appl. Mech. 1961; 28: 112-116.

[23] Coman, CD. Asymptotic approximations for pure bending of thin cylindrical shells. Z. Angew. Math. Phys. 2017; 68:82. 
[24] Coman, CD. Bifurcation instabilities in finite bending of circular cylindrical shells. Int. J. Eng. Sci. 2017; 119: 249-264.

[25] Troger, H, Steindl, A. Nonlinear Stability and Bifurcation Theory. Wien: Springer-Verlag, 1991.

[26] Batdorf, SB. A simplified method of elastic-stability analysis for thin cylindrical shells. Report No. 874, Langley Memorial Aeronautical Laboratory, Langley Field, Va. 1947.

[27] Novozhilov, VV. The Theory of Thin Shells (second edition). Gröningen: P. Noordhof Ltd., 1964.

[28] Sanders, JL. Nonlinear theories for thin shells. Quart. Appl. Math. 1963; 21: 21-36.

[29] Coman, CD, Bassom, AP. Wrinkling of pre-stressed annular thin films under azimuthal shearing. Math. Mech. Solids 2008; 13: 513-531.

[30] Libai, A, Durban, D. Buckling of cylindrical shells subjected to non-uniform axial loads. ASME J. Appl. Mech. 1977; 44: 714-720.

[31] Kantorovich, LV, Krylov, VL. Approximate Methods of Higher Analysis. New York: Interscience, 1958.

[32] Kerr, AD. An extension of the Kantorovich method. Quart. Appl. Math. 1968; 26: 219-229.

[33] Kerr, AD, Alexander, H. An application of the extended Kantorovich method to the stress analysis of a clamped rectangular plate. Acta Mech. 1968; 6: 180-196.

[34] Kerr, AD. An extended Kantorovich method for the solution of eigenvalue problems. Int. J. Solids Struct. 1969; 5: 559-572. 